\title{
ProCACD: A computer-aided versatile tool for process control
}

\author{
Tula, Anjan K.; Wang, Jialiang; Chen, Xi; Mansouri, Seyed Soheil; Gani, Rafiqul
}

\section{Published in:}

Computers \& Chemical Engineering

Link to article, DOI:

10.1016/j.compchemeng.2020.106771

Publication date:

2020

Document Version

Peer reviewed version

Link back to DTU Orbit

\section{Citation $(A P A)$ :}

Tula, A. K., Wang, J., Chen, X., Mansouri, S. S., \& Gani, R. (2020). ProCACD: A computer-aided versatile tool for process control. Computers \& Chemical Engineering, 136, [106771].

https://doi.org/10.1016/j.compchemeng.2020.106771

\section{General rights}

Copyright and moral rights for the publications made accessible in the public portal are retained by the authors and/or other copyright owners and it is a condition of accessing publications that users recognise and abide by the legal requirements associated with these rights.

- Users may download and print one copy of any publication from the public portal for the purpose of private study or research.

- You may not further distribute the material or use it for any profit-making activity or commercial gain

- You may freely distribute the URL identifying the publication in the public portal 


\section{Journal Pre-proof}

ProCACD: A computer-aided versatile tool for process control

Anjan K. Tula, Jialiang Wang, Xi Chen, Seyed Soheil Mansouri , Rafiqul Gani

PII:

DOI:

Reference:

To appear in:

Received date:

Revised date:

Accepted date:
S0098-1354(19)31107-X

https://doi.org/10.1016/j.compchemeng.2020.10677

CACE 106771

\section{Computers and Chemical Engineering}

23 October 2019

13 January 2020

5 February 2020

Please cite this article as: Anjan K. Tula, Jialiang Wang, Xi Chen, Seyed Soheil Mansouri, Rafiqul Gani , ProCACD: A computer-aided versatile tool for process control, Computers and Chemical Engineering (2020), doi: https://doi.org/10.1016/j.compchemeng.2020.106771

This is a PDF file of an article that has undergone enhancements after acceptance, such as the addition of a cover page and metadata, and formatting for readability, but it is not yet the definitive version of record. This version will undergo additional copyediting, typesetting and review before it is published in its final form, but we are providing this version to give early visibility of the article. Please note that, during the production process, errors may be discovered which could affect the content, and all legal disclaimers that apply to the journal pertain.

(C) 2020 Published by Elsevier Ltd. 
Highlights

- An integrated software, ProCACD, is developed for process design-control.

- ProCACD includes model library, design methods, analysis methods and integrated tools.

- Analysis of process with open-loop or closed-loop controllers can be conducted.

- Optimal controller design and analysis are presented.

- Simultaneous process design-control methods are developed in ProCACD. 


\title{
ProCACD: A computer-aided versatile tool for process control
}

\author{
Anjan K. Tula ${ }^{\mathrm{a}}$, Jialiang Wang ${ }^{\mathrm{a}}$, Xi Chen ${ }^{\mathrm{a}^{*}}$, Seyed Soheil Mansouri ${ }^{\mathrm{b}}$, Rafiqul Gani ${ }^{\mathrm{c}^{*}}$ \\ ${ }^{a}$ College of Control Science and Engineering, Zhejiang University, Hangzhou-310027, China. \\ ${ }^{b}$ Process and Systems Engineering Center, Department of Chemical and Biochemical Engineering, \\ Technical University of Denmark, Kgs. Lyngby DK-2800, Denmark. \\ ${ }^{c}$ PSE for SPEED, Skyttemosen 6, Allerod, DK-3450, Denmark.
}

*Corresponding authors: Xi Chen (xichen@zju.edu.cn) \& Rafiqul Gani (rgani2018@gmail.com)

\begin{abstract}
A number of powerful tools have been developed for process simulation, optimization, and design. However, the current software in Process Systems Engineering is mainly focused on processes without consideration of controller design or validation. Therefore, there is always a demand for integrated platforms for solving various process design and control problems. In this paper, an integrated software, ProCACD, developed for solution and analysis of control related problems is presented. Embedded within the software architecture are process design methods, analysis methods, integrated tools, and a library of process models that allow users to study a wide range of problems. With ProCACD, users can solve problems involving generation of process models, steady-state and/or dynamic simulations, analysis of process dynamics in open-loop and/or closed-loop, design of the controller structure, and solving integrated process-control design problems. The application of the software-tool is highlighted through four examples that illustrate the use of features available in ProCACD.
\end{abstract}

Keywords: Process simulation; process design; controller design; integrated platform; computer-Aided software; ProCACD. 


\section{Introduction}

Nowadays sustainable and efficient processes are needed by the chemical and related industries to address the grand challenges of resource depletion, energy consumption and environmental impacts. Therefore, design, development and operation of efficient processes are important for the industry to remain competitive. During the past decades, many computer-aided software tools have been developed to address different aspects of process development. For example, GAMS (GAMS Development Corporation, 2019), ASCEND (Piela, 1990), MoT (Sales-Cruz and Gani, 2003), etc., for process modeling; AspenOne (Aspen Technology, 2019a), Hysys (Aspen Technology, 2019b), ProII (SimSci, 2018), gPROMS (Process Systems Enterprise, 2018), etc., for process simulation and optimization; and ProCAFD (Tula et al., 2017) for process synthesis and design. These tools have made major contributions to the Process Systems Engineering (PSE) community and each of them have their particular features as well as advantages and deficiencies. Although process control is a very important branch of PSE, its development is not very well coordinated with the developments in other core areas of PSE. One important reason could be that process simulation and optimization usually employ detailed rigorous models based on mass and energy balances, and, many of these applications are offline. On the other hand, in process control, many of the applications are online and data-driven. Moreover there is a scarcity of computer aided platforms that can address complex control problems related to integrated design, control and scheduling (Maurmaier, 2008; Pistikopoulos et al., 2015).

Though a number of powerful computer-aided tools have been developed in the past decades, there is still a lack of tools, which can combine process simulation, design, optimization, and control in a systematic and efficient way, especially rigorous model-based process design, optimization and control. The objective of process design is to identify the best sequence of tasks/operations that need to be performed, and the corresponding design of the operation/equipment, their configuration, mass/energy flows, etc. (Tula et al., 2015). Process control aims to maintain the process at the desired operating points (set points) subject to disturbances (Gollapalli et al., 2000; Yuan et al., 2012). Traditionally, process design and control problems have been treated as independent problems (Nelson et al., 1989) and solved separately, where the process is designed first to achieve the desired design objectives and then for a given solution of the design, the operability and control aspects are analysed and resolved to obtain the controller design to maintain the process at the desired operating level within the limits of 
the design constraints. This approach, however, does not always guarantee optimal solutions and may even point out that what is good for design may not be good for control (Seferlis and Georgiadis, 2004).

Several tools have been developed to address different sub-problems in the areas of process design and control. In the area of process control, CONSYD (Holt et al., 1987) is probably one of the first computer-aided tool developed for interactive controller design and simulation. It is based on individually callable FORTRAN programs that are connected by common file types that include plant models and controller models. This tool is mainly used for design of control structures and analysis through dynamic simulations. Later in the 1990's Cooper developed PICLES to simulate process dynamics and for solution of control problems (Cooper, 1996), which became an improved commercial software called ControlStation (www.controlstation.com). However, the most widely used tools to address problems in process control are still Matlab (Mathworks, 2019a) and Simulink (Mathworks, $2019 b$ ), with a number of toolboxes that can be used for system identification, controller tuning and analysis. In order to use the specific functions of these toolboxes the user needs to write programming scripts to send data and plot the results. Chemcad dynamics (Chemstations, 2019) and Aspen dynamics (Aspen Technology, 2019c) are other popular tools that are used for dynamic flowsheet simulations and analysis based on simulations with embedded controllers. Some other tools in this area are PITOPS (Howes et al., 2016), SIMCET (Howes et al., 2016), PID Control laboratory (PID Control Laboratory, n.d.) etc., which are used mainly for PID tuning, loop optimization, dynamics identification (transfer function identification) and operator training. Though a number of powerful tools have been developed in the past decades, the effort has been concentrated in solving a few core sub-problems related to process design and/or control. In this regard there is still a lack of an integrated platform that can combine the process simulation, design, optimization, and control functions in a flexible, versatile, efficient and systematic manner. With this integrated platform users should be able to easily build a process model based on a generic formulation, conduct the steady-state and/or dynamic simulation, analyze the process dynamics in open-loop or closed-loop with controllers, configure and improve the controller design, and eventually perform integrated process design-control studies.

The objective of this paper is to introduce a flexible, reliable and efficient model-based system for solution of a wide range of control related problems. Established modelling, design and control related 
methods and associated tools are integrated through a multi-layered component-based software architecture. For various control related problems, the software retrieves a corresponding in-house problem specific work-flow and the needed models, data, design methods, including methods for controller pairing and tuning, etc., to solve the problem without the need for additional tools or programming and/or data transfer. This paper provides details of the architecture of the software tool (ProCACD) in section 2, the implemented and/or adopted methods and tools in section 3 and four illustrative examples to highlight its applicability.

\section{Overview of ProCACD}

Efficient, reliable and robust control of chemical and/or biochemical (C\&B) processes needs a very good understanding of the operated domain system. Assuming a validated model of the system is available, a model-based control system can be designed, tested and implemented. Depending on the application range of the available model, aspects of process design can also be included. In this section, first a generic mathematical formulation of the process (system) control problem that also incorporates some aspects of process design is presented, followed by a presentation of the architecture of ProCACD, a software tool that has been developed to solve a wide range of process control related problems, including integrated process design and control.

\subsection{Generic Problem Formulation}

The generic mathematical formulation of the process design-control problem is given by Equations 1-5.

$$
O b j=\left\{S_{d}+S_{c}\right\}
$$

s.t:

$\frac{d x}{d t}=f(x, y, u, d, \theta, Y, t)$

$h(x, y, u, d, \theta, Y, t)=0$

$g(u, x, y) \geq 0$

$y_{c}=f_{c}\left(u_{c}, Y_{c}, \theta_{c}\right)$ 
In the above equations, Equation 1 represents the objective function that needs to be minimized or maximized and may include multiple terms accounting for performance of the process related to design and/or control. Both design criteria $\left(S_{d}\right)$ and the control criteria $\left(S_{c}\right)$ may focus on issues such as economics, energy consumption, waste, operability and flexibility. The process model (Equation 2) satisfies the conservation of mass, energy \& momentum. Here $x$ represents the system states and $y$ represent the system outputs along with algebraic variables. $u$ is the set of design variables (for process design $u_{d}$ ) and/or the set of manipulated variables (for controller design $u_{c}$ ). $d$ is the set of disturbance variables, $\theta$ is the set of design $\left(\theta_{d}\right)$ and controller $\left(\theta_{c}\right)$ parameters. $Y$ represents the decision variables for process design $\left(Y_{d}\right)$ and controller design $\left(Y_{c}\right)$ while $t$ is the independent variable (usually time, for the dynamic model). Note that if the left-hand side of Equation 2 is zero, it represents a steady state model of the process. Equation (3) represents constitutive equations which relate the algebraic constitutive variables (properties) to the measurable (intensive) process variables (such as temperature, pressure and/or compositions), or the output equations, which relate the output variables to the state variables. Equation (4) represent sets of operational constraints (such as product purity, concentration in a specific stream) that must be satisfied for feasible operation and/or design. These constraints can be linear or non-linear, depending on the equations that represent them. Equation (5) describes the control law, where is the set of binary decision variables for controller structure selection (indicates the pairing of a controlled variable with a manipulated variable) and represents a set of the corresponding controller parameters; $y_{c}$ and $u_{c}$ represent the set of controlled variables and manipulated variables, respectively. Since the models here can be nonlinear and the variables involved can be integers as well as real, the generic problem defined above could represent in a complex mixed integer nonlinear programming (MINLP) formulation for steady states or mixed integer dynamic optimization (MIDO) formulation for dynamic states. Several types of process control related problems can be generated from the generic mathematical formulation, as listed in Table 1. 
Table 1: Examples of process design-control problems

\begin{tabular}{|c|c|c|}
\hline Problem type & Model equations involved & Action \\
\hline $\begin{array}{l}\text { Model } \\
\text { generation }\end{array}$ & $\begin{array}{l}\text { Equations } 2 \& 3 \text { for the process model to be } \\
\text { studied }\end{array}$ & $\begin{array}{l}\text { Develop and validate the required } \\
\text { process model (Equations } 2 \& 3 \text { ) }\end{array}$ \\
\hline $\begin{array}{l}\text { Open-loop } \\
\text { simulation }\end{array}$ & Equations ( $2 \& 3)$ for process simulation. & $\begin{array}{l}\text { Steady state or dynamic forms of } \\
\text { Equation } 2 \text { and Equation } 3 \text { are used } \\
\text { to simulate behavior of systems } \\
\text { inputs and outputs for given values of } \\
\text { all other variables }\end{array}$ \\
\hline $\begin{array}{l}\text { Controller } \\
\text { design }\end{array}$ & $\begin{array}{l}\text { Equation (1) (the objective function should } \\
\text { be based on minimizing control } \\
\text { performance), Equations }(2,3,4) \text { for process } \\
\text { calculations and process related constraints. } \\
\text { Equation }(5) \text { with different control } \\
\text { parameters. }\end{array}$ & $\begin{array}{l}\text { The optimal values of the controller } \\
\text { parameters }\left(\theta_{c}\right) \text { are obtained usually } \\
\text { using linear process control models }\end{array}$ \\
\hline $\begin{array}{l}\text { Closed-loop } \\
\text { simulation }\end{array}$ & $\begin{array}{l}\text { Equations }(2 \& 3) \text { for dynamic simulation. } \\
\text { Equation (5) with fixed control structure and } \\
\text { parameters. }\end{array}$ & $\begin{array}{l}\text { Only the dynamic form of Equation } 2 \\
\text { is used together with Equations } 3 \& 5 \\
\text { to study the transient responses } \\
\text { subject to feed disturbances or set- } \\
\text { point change. }\end{array}$ \\
\hline $\begin{array}{l}\text { Controller } \\
\text { performance } \\
\text { analysis (for } \\
\text { different } \\
\text { control } \\
\text { strategies) } \\
\end{array}$ & $\begin{array}{l}\text { Equations ( } 2 \text { \& } 3) \text { for dynamic simulation, } \\
\text { Equation (4) for process related constraints. } \\
\text { Equation (5) with different control structure } \\
\text { and parameters. Controller structure } \\
\text { screening based on ITSE for different } \\
\text { configurations. }\end{array}$ & $\begin{array}{l}\text { Repeat the closed-loop simulations } \\
\text { for different controller pairings } \\
\text { and/or designs }\end{array}$ \\
\hline $\begin{array}{l}\text { Design \& } \\
\text { control }\end{array}$ & $\begin{array}{l}\text { Equation (1) (the objective function should } \\
\text { be based on optimizing design decisions } \\
\text { only), Equations }(2,3,4) \text { for process } \\
\text { calculations and process related constraints. } \\
\text { Equation (1) (the objective function should } \\
\text { be based on optimizing design decisions and } \\
\text { control performance), Equation }(2) \text { for } \\
\text { dynamic simulation, Equations }(3,4) \text { for } \\
\text { process calculations and process related } \\
\text { constraints. Equation }(5) \text { with different } \\
\text { control parameters. }\end{array}$ & $\begin{array}{l}\text { The optimal values for } u_{d} \text { and } \theta_{d} \text { are } \\
\text { obtained subject to desired values of } \\
x \text { and } y \text { plus other constraints } \\
\text { The optimal values for }\left(u_{c} u_{d}\right) \text { and } \\
\left(\theta_{d}\right) \text { and are obtained subject to } \\
\text { desired values of } x \text { and } y \text { plus other } \\
\text { constraints }\end{array}$ \\
\hline
\end{tabular}




\subsection{Architecture (Framework) of ProCACD}

From the various specific control related problem formulations, it is clear that there is a need for a platform (software) that can integrate different models, associated data, solver requirements, etc., for the specific problem of interest. The ProCACD software tool, which can support the control related problems listed in Table 1, has been developed based on a component-based software architecture (Tula et al., 2018). This is also a multi-layered architecture, where tailor-made problem specific software tools are generated by assembling different software components that are taken from a database of components. This approach emphasizes separating the functionality of a software into independent, interchangeable components, such that each component contains the necessary tools to execute one aspect of the desired functionality. ProCACD is developed in visual C\# environment using the concepts of object-oriented programming. Figure 1 shows the architecture of ProCACD in terms of components and interaction between the main interface and the components by the directional connections, which indicate the dataflow. As highlighted in the figure, there are four principal components (model library, design methods, analysis methods and integrated tools) at the outer-layer. In the next layer, are the available classes of components. In the next layer (not shown in the figure), the available sub-classes of the components are placed. Finally, in the innermost layer (not shown in the figure), the specific contents of the associated component sub-class are stored, which can be retrieved as and when necessary. For example, the model library component includes a collection of models, which are classified in terms of steady state, dynamic, state space and transfer functions. Within each class (not shown in the figure), a collection of models that have been developed and verified a priori are stored. Similarly, the "integrated tools" component provide the available links to classes of external tools, the "design methods" component provide the available classes of methods for design (including simulation), and the "analysis methods" component provide the available classes of methods for analysis. This architecture allows a systematic and efficient way for accessing the needed models, tools, data, solvers, etc., to solve a specific control related problem through the associated inhouse work-flow for each control related problem (see Figure 2). Also, this architecture allows easy maintenance and extension. For example, a specific model or method of a component could be easily replaced by a newer and better version. Or, A new model or method could easily be added to the existing list. Or, a new control related problem with its corresponding work-flow implementation, would be able to extract the needed tools directly from the components. 
By following the directions in the general work-flow diagram highlighted in Figure 2, the component needs for a specific control related problem listed in Table 1 is generated. After the component needs are established and the specific classes of components are retrieved, ProCACD guides the user through the steps of the individual problem work-flows that are available for different control related problems. These individual work-flows (methods) and associated tools for each control related problem covered by ProCACD are described in the next section. For example, to analyze the controller performance of a specified system, Figure 2 is used the trace the path of the tools (grey boxes) needed. First a model is selected, then, following the "analysis" path, "controller performance" and then "frequency domain analysis" are selected. With this information, ProCACD retrieves the individual work-flows (see section 3) for each selected tool and the required components from the stored contents under each component.

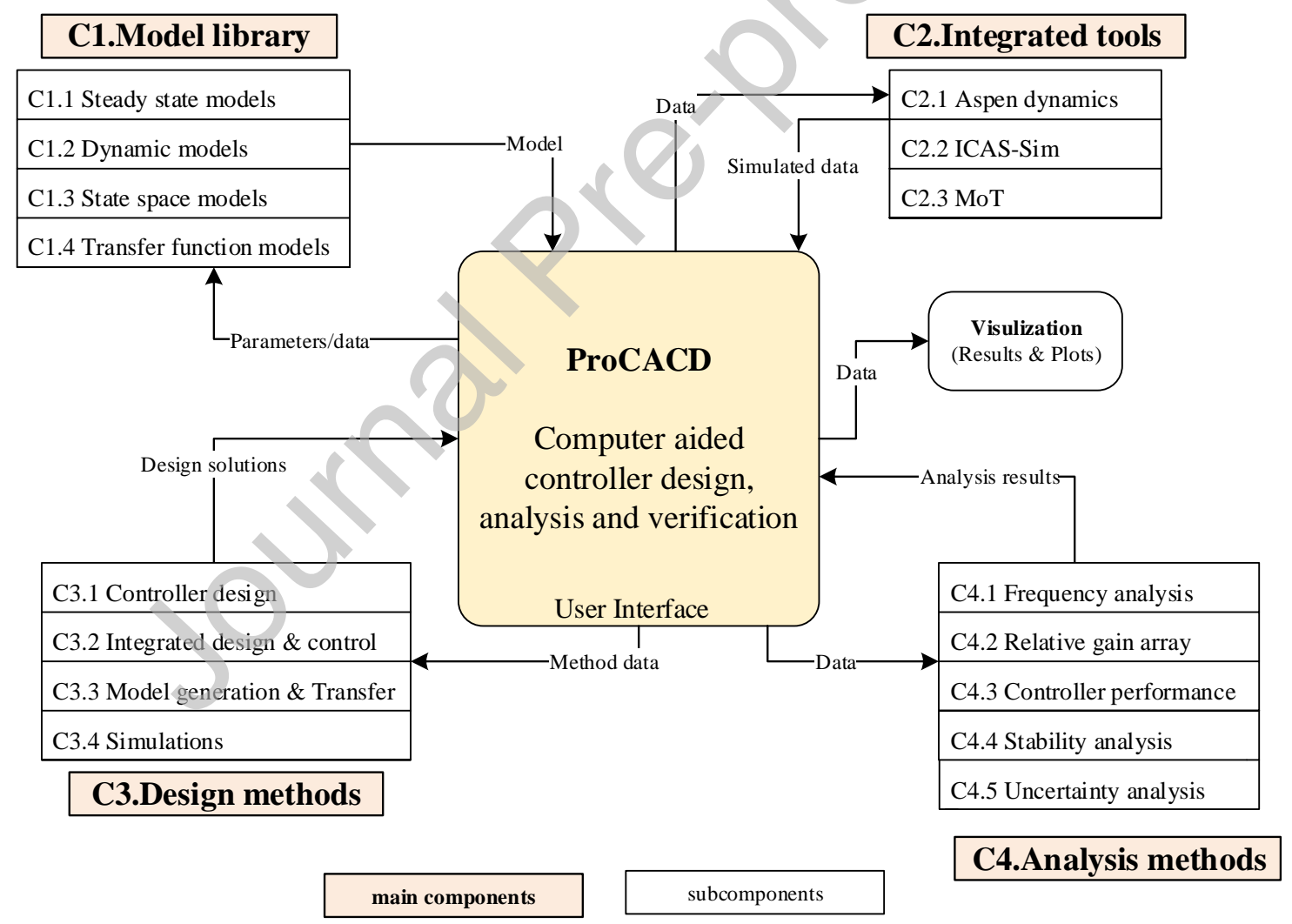

Figure 1: Architecture of ProCACD 


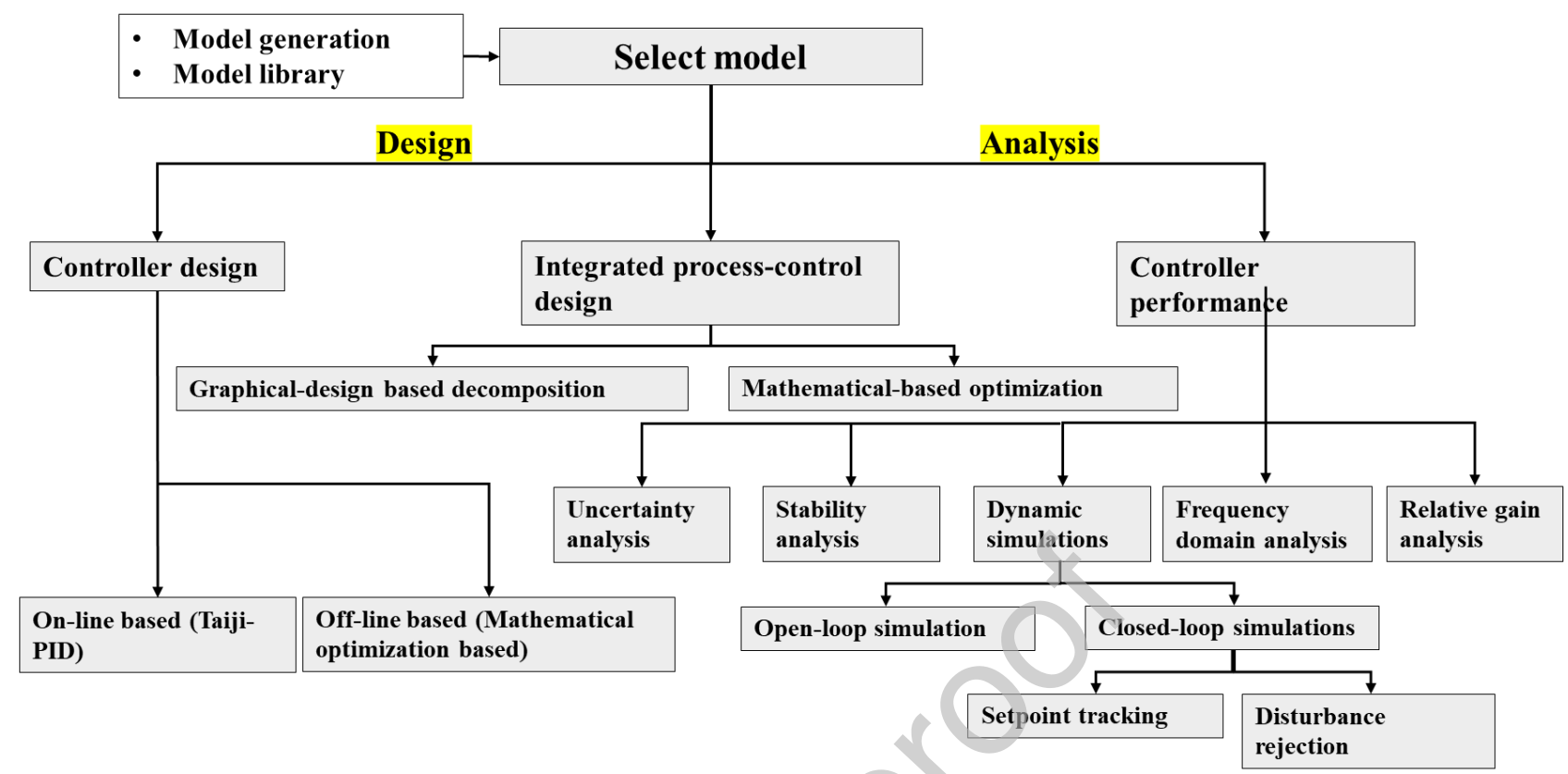

Figure 2: General work-flow for use of ProCACD

\section{Methods and Associated Tools of ProCACD}

\subsection{Model Generation \& Model Library}

ProCACD can be used to develop models for a system for which the needed model is not available in the model library. In order to generate these models ProCACD is linked with MoT, which is an inhouse modelling tool-box (Sales-Cruz and Gani, 2003), where model construction is based on the use of a modelling template as shown in Figure 3. The modelling template guides the user to organize the model equations as balance equations, constitutive relations and connection and/or conditional equations, based on the modelling hierarchy proposed by Cameron and Gani (2011). Within each type of equations, multiple versions of model equations can be stored and retrieved by the use of binary variables to generate different problem-specific models (Fedorova et al., 2015). Figure 3 highlights a generic model template where the four types of model equations and what they generally represent of the system are shown. Other options to develop a model and insert to the simulation system, such as using Matlab (Mathworks, 2019a) or Aspen-Modeler (Aspen Technology, 2019d) could also be used. 


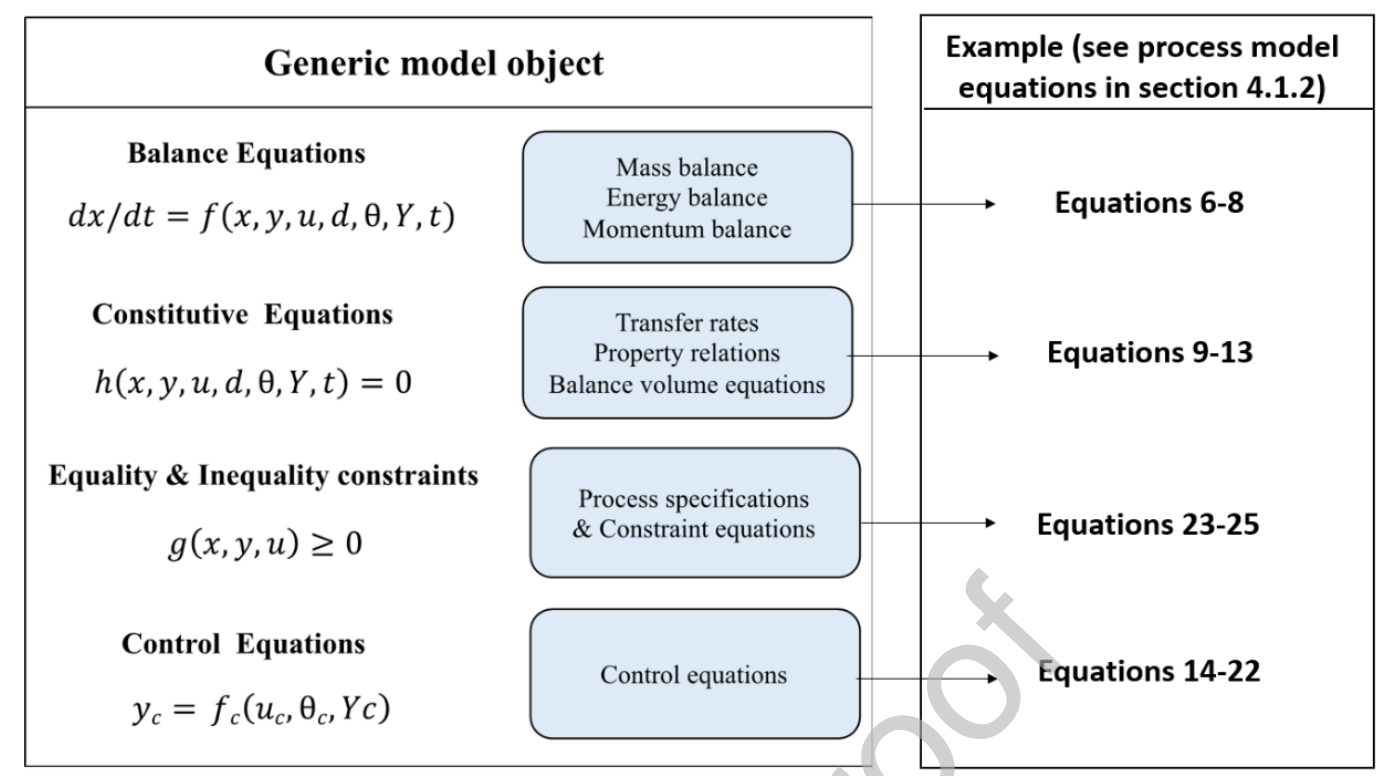

Figure 3: Generic model template

ProCACD contains a well-developed library of process models covering a wide range of unit operations and a selected set of process flowsheets. Section S1 of supplementary material gives a selected list of process models available in the model library of ProCACD, where all the models are used through MoT.

\subsection{Simulations}

This problem needs to be solved when a) to study the influence of process design variables $(u)$ and parameters $(\theta)$; b) to study the effect of variables such as set-point change or feed disturbances in openloop transient responses; c) to evaluate the performance of a controller design (pairing as well as controller parameters); d) to develop the model-based optimal control or integrated design-control. In each case, model-based simulations need to be performed. The work-flow for these problems is briefly described as it is a standard option that is available in all process simulators as well as in MoT. The work-flow consists of the following steps: retrieve (select) the needed model from the model library of the selected process simulator or from ProCACD; specify all the input variables; run the process simulator using the simulation option of the selected simulator or from ProCACD (through available links to external simulators). If the needed model is not available, then first the process model would need to be developed. 


\subsection{Controller Design}

A typical control system is shown in Figure 4, where the process block is represented by a process model and its associated simulation tools. The controller block includes design of various types of controllers. Nowadays, proportional-integral-derivative (PID) control and model predictive control (MPC) are dominant in industry. MPC is an advanced control strategy that works efficiently for a large number of multivariable systems. However, due to advantages of low cost and easy implementation and maintenance, PID controllers still occupy more than $90 \%$ in practice. In this paper, the development of ProCACD focuses on PID controllers, although, application of specific MPC is also possible.

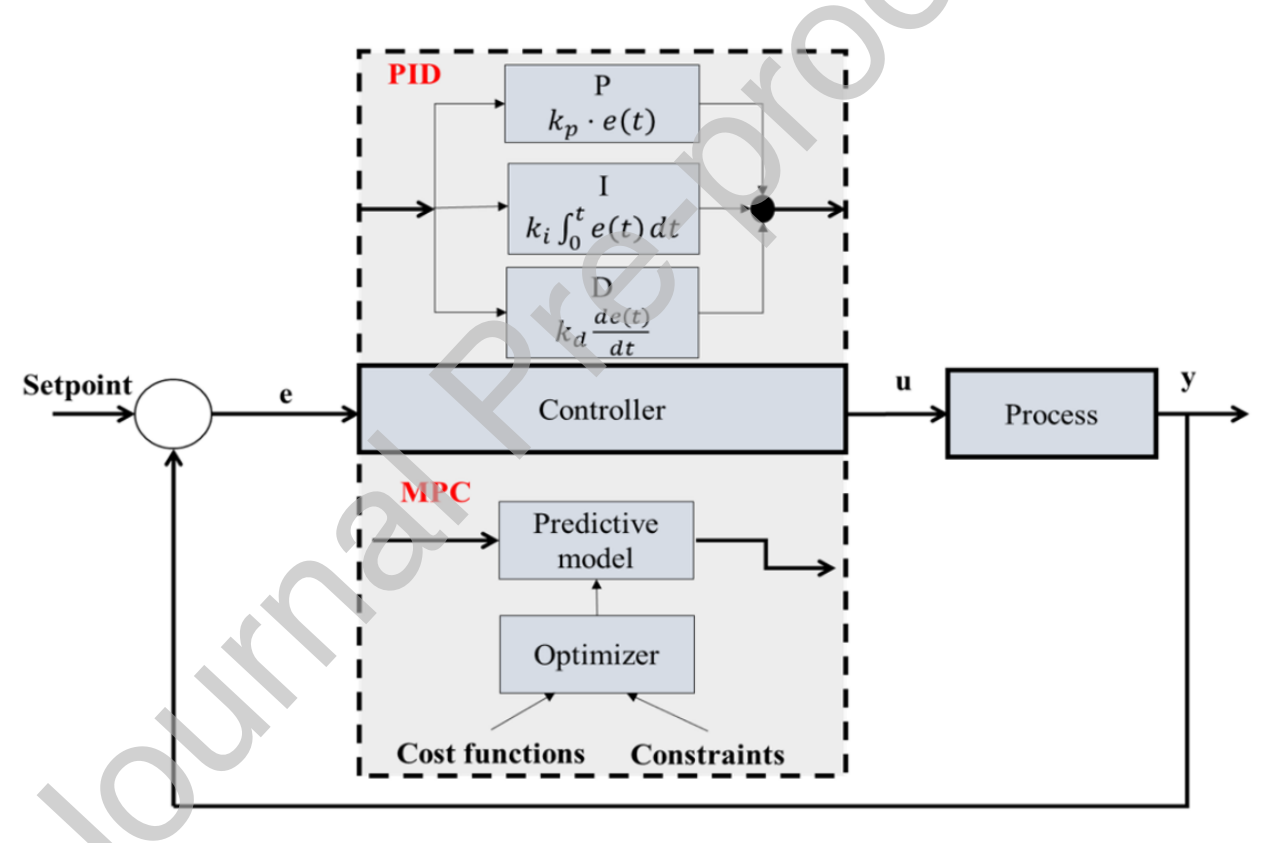

Figure 4: Flow-diagram for model-based control system design

In a manufacturing setup, a good controller design is very important to guarantee a desired response of the process system. Problems involving design of optimal controllers can be solved using ProCACD. The optimal controller design is achieved, in ProCACD, in two sequential steps involving controller pairing and controller tuning, which can also be performed simultaneously. It should be noted that many methods for controller pairing and tuning exist, and, only those with which the authors are 
familiar and have experience with, have been implemented in this first version of ProCACD. The architecture of ProCACD, however, is flexible and will allow the addition of user-added work-flows based on other methods.

\subsubsection{Controller pairing}

The main objective of controller pairing is to select optimal pair of available controlled variables $(\underline{\mathrm{CV}})$

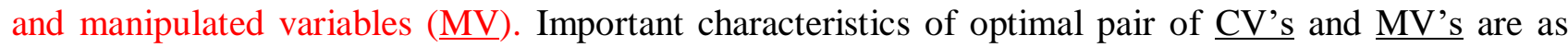
follows:

a) The optimal sensitivity of controlled variable with respect to disturbances should be low.

b) The sensitivity of controlled variables with respect to manipulated variables should be high.

ProCACD uses the relative gain array method to find the optimal pair of controlled variables and manipulated variables (see also integrated design and control in section 3.5 where graphical techniques are used to near optimal design-controller structures). The work-flow is shown in Figure 5. In step 1, ProCACD uses all the available CV-MV pairs to generate step response data by setting the process model to open-loop setting. In step 2, this simulation data is used to generate the corresponding transfer function models through the TAIJI-PID controller design tool available online (www.taijipid.com). In step 3, ProCACD uses the transfer function model to generate relative gain array matrix for all the CVMV pairs. Finally, the pair having the values of the relative gain array close to unity on the diagonal (and with negligible off-diagonal elements) are selected. Note that ProCACD can also use the rigorous dynamic models in open-loop simulation mode to generate the transfer function matrix and the corresponding relative gain array. 


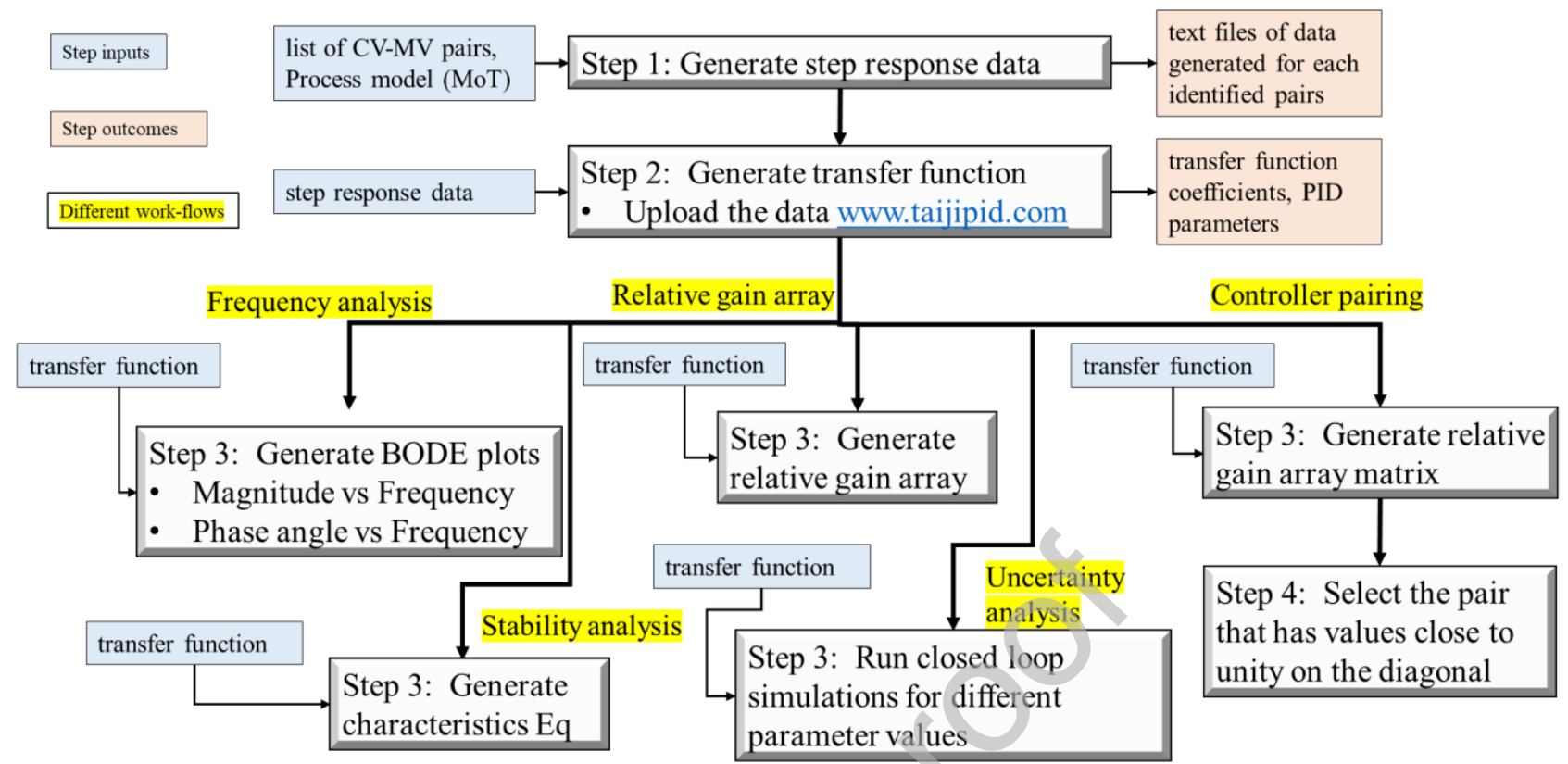

Figure 5: work-flow for optimal controller pairing, frequency analysis, relative gain array, stability analysis and uncertainty analysis

\subsubsection{Controller tuning}

This is one of the most important steps in controller design and has direct effect on the performance of the controller due to disturbances in feed or set-point. Given the controller structure and control law, the controller parameters govern the extent and rate of the changes that will make to the process. Taking a PID controller, for example, the P (proportional), I (integral), and D (derivative) parameters decide the control action and thereby, its performance. If the controller parameters are not properly set, the process may response very slack, or even worse, the operation may become unstable. On the other hand, a well-tuned controller can maintain the process operation react along a desired trajectory. Therefore, it is very important to find the optimal values for the controller parameters. In ProCACD, parameter tuning for PID controllers have been developed with two methods.

I. On-line tuning (Taiji-PID design method): In this method there are three steps as shown in Figure 6 for controller tuning. In step 1, the identified optimal pair of controlled and manipulated variables and the process model (MoT) are used by ProCACD to generate the 
open-loop response data. This is done by applying step changes on the manipulated variable and recording the response. In step 2, ProCACD connects to the Tai-ji PID method for controller design through a web-API ("Taiji-PID," 2019) deployed in public cloud (www.taijipid.com). The step response data is uploaded to the Taiji-PID website, which in return sends the transfer function along with the tuned PID parameters. Step 3 (verify control) is discussed under "analysis" (section 3.4.3).

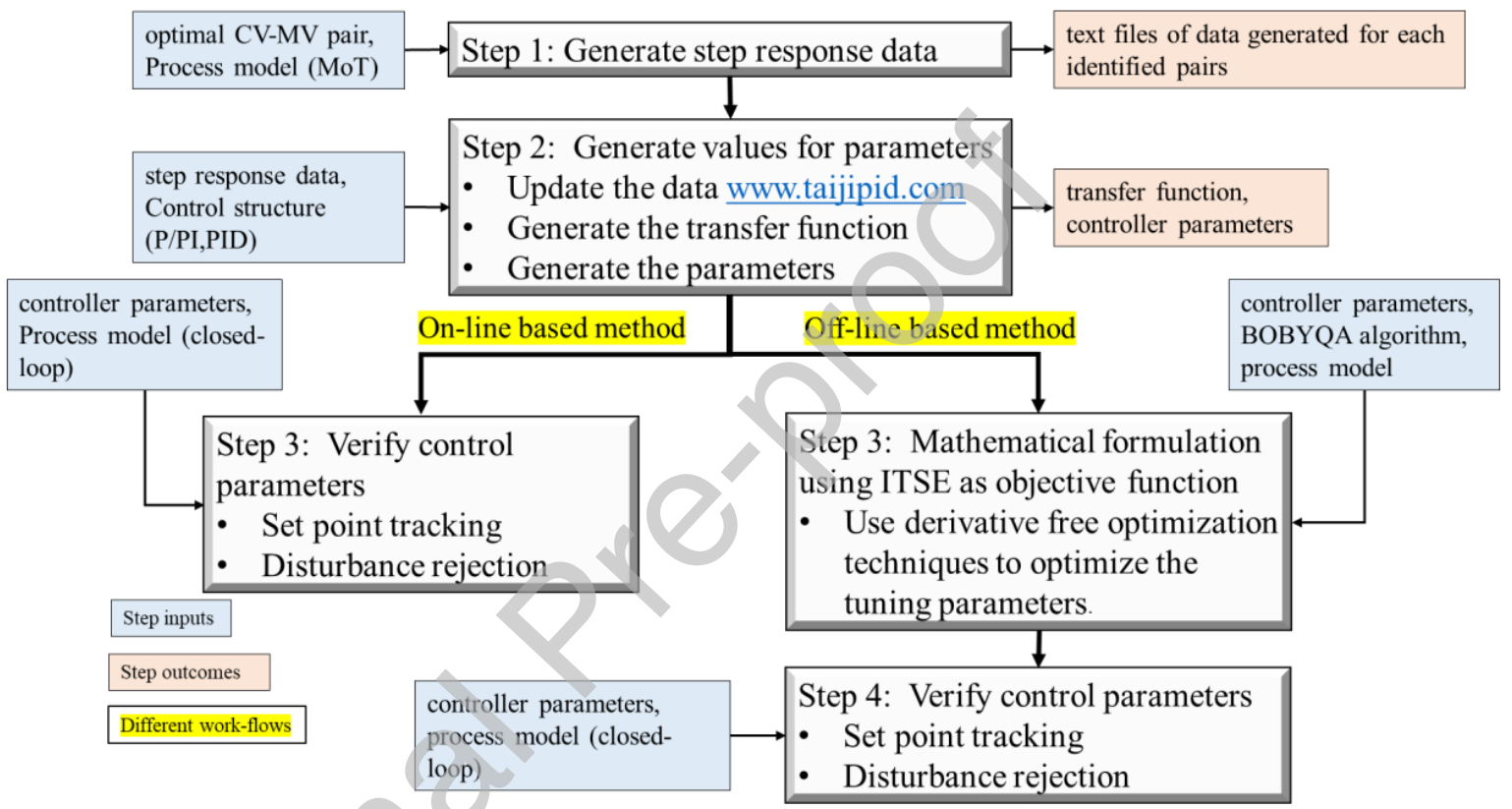

Figure 6: Work-flow for controller tuning (on-line method / off-line based method)

II. Off-line tuning (Optimization based method): In this method, as shown in Figure 6, there are 4 steps. In step 1, the identified optimal pair of controlled and manipulated variables and the process model (MoT) is used by ProCACD to generate the open-loop response data. In step 2, ProCACD generates the initial values of the controller parameters by regressing the transfer function parameters (Pernebo and Silverman, 1982) using the data generated in step 1. In step 3, the process model is updated with these (initial) controller parameters and set to closed-loop simulation mode. Based on this closed-loop configuration, ProCACD further tunes the PID controller parameters by adding a numerical solver for optimization as an outer-loop, where an objective function minimizes (or maximizes) a specific performance index. For the outer-loop 
optimization, a gradient-free optimization technique, BOBYQA (Powell, 2009) has been implemented in ProCACD. Also, other solvers are available through MoT. Step 4 (verify control) is discussed under "analysis" (section 3.4.3).

\subsection{Controller Analysis}

In addition to steady state and dynamic (open-loop and closed-loop) simulations for analysis of the process behavior and better understanding of the process, additional control related analysis tools have also been implemented in ProCACD. To integrate the process design-control, ProCACD can analyze the process design issues together with control performance with different types of process models. In this section the analysis methods currently embedded in ProCACD are presented along with their workflows.

\subsubsection{Frequency domain analysis}

Predicting the future behavior of a process is key to the analysis of feedback control systems. To capture the process dynamics, it is essential and convenient to characterize the process in the frequency domain. The frequency analysis shows how the process reacts for different frequency signals, for example, by varying the frequency of the input signal over a certain range and evaluating the resulting transient response. The analysis in ProCACD is given as BODE plots, which can be used to determine the amplitude and phase of the output that results when the process is driven by a sinusoidal input with a particular frequency. Figure 5 shows the work-flow for generating frequency analysis plots in ProCACD. Steps 1 and 2 are the same as explained in section 3.3.1. In step 3, the transfer function information is used by ProCACD to generate the frequency analysis plots.

\subsubsection{Relative gain array}

The relative gain array (RGA) analysis is a widely-used classical method for determining the best input-output pairings for multivariable process control systems. Relative gains for the diagonal elements close to 1 and for off-diagonal elements close to 0 indicate good selection of variables, while values of 0.5 for off-diagonal elements indicate strong interactions. In general, the variables closest to 1 are paired to keep the interactions to minimum. By integrating the process design-control, ProCACD 
allows the user to exploit the developed process model to identify the best pairing for process controller design. Figure 5 shows the workflow for generation of relative gain array and analyze the pairing in ProCACD. Steps 1 and 2 are the same as explained in section 3.3.1. In step 3, the transfer function information generated in step 2 is used to calculate relative gain matrix for the selected CV-MV pair. It should be noted that, for some problems, RGA is not suitable. Other techniques like decoupling and MPC may be more appropriate, which have not been implemented yet in ProCACD.

\subsubsection{Controller performance}

As an integrated platform, ProCACD can analyze the control performance for a user-defined process with a specified controller design, which is very important to synthesize the controller and its eventual process implementation. This method is used to test the performance of controllers using transient responses from closed-loop simulations due to changes in input variables. There are two important criteria based on which performances of the controllers are evaluated currently in ProCACD: the ability of the controller to track the set point change and the ability of the controller to reject the disturbance in the inputs. Figure 7 shows the work-flow for controller performance in ProCACD. In step 1, the control structure with parameters are loaded into ProCACD. Based on the user selection (disturbance rejection/set point tracking), the dynamic process model is initialized with the uploaded parameters and is set to closed-loop simulation mode. In step 2, based on the choice in step 1 a step change is made to input (disturbance) or set point and simulations are performed for a certain period. In step 3, the closed-loop response data is used to generate the combined plot in ProCACD. From this comparison of plots, the best control structure/parameters can be identified from the list of different controllers and their performance index. 


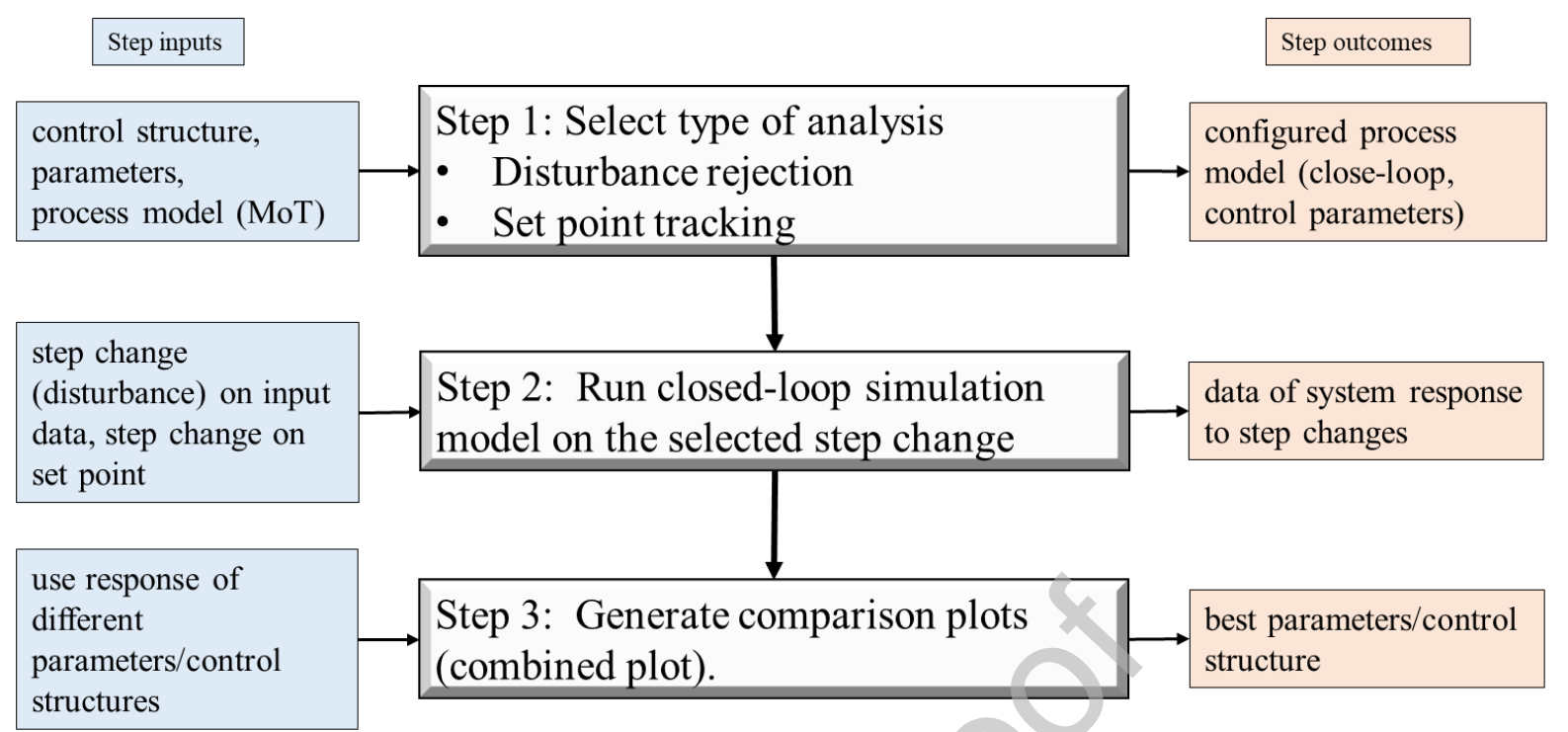

Figure 7: Work-flow for controller performance

\subsubsection{Stability analysis}

Stability analysis is key to application of process controllers. ProCACD allows the user to analyze both open-loop and closed-loop stability of the loops. The key method for stability analysis currently available in ProCACD is the Routh stability criterion based on characteristic equations of a system. Figure 5 shows the work-flow for stability analysis in ProCACD. Steps 1 and 2 are the same as explained in section 3.3.1. In step 3, the transfer function information is used by ProCACD to generate characteristic equations of the loops. For closed-loop, the controller parameter information is also needed. ProCACD judges the stability of the loops with an embedded Routh stability criterion algorithm based on coefficients of the characteristic equations.

\subsubsection{Uncertainty analysis}

Uncertainty analysis is important to evaluate the robustness of the closed-loop process. ProCACD allows the user to do uncertainty analysis based on closed-loop simulation. In this analysis users can change or give a range for some parameters of the model and do closed-loop simulation under the new condition. Here ProCACD generates a combined plot to see the effect of the parameter values on the controller performance. More features will be considered in future. 


\subsection{Integrated Process Design and Control}

Two integrated design-control approaches have been implemented in ProCACD as initial test cases, based on which other methods will be added in future.

\subsubsection{Graphical design-based decomposition approach}

This approach is based on locating the design target using graphical methods such as attainable regions for reactors ((Glasser and Hildebrandt, 1997; Hildebrandt and Glasser, 1990; Horn.F, 1964) and driving force-based design for separation processes (Gani and Bek-Pedersen, 2001). In both cases, the objective is to design and operate (and therefore control) at the highest attainable point for reactions or at the maximum available driving force for separations, especially, distillation columns. (AlvaradoMorales et al., 2010; Hamid et al., 2010; Mansouri et al., 2016b, 2016a) showed the application in reactive distillation columns. It was observed that designing the process at the maximum point of driving force diagrams (for distillation columns, with or without reactions), resulted in minimum operating cost, lowest energy requirements, fast controller response and better controller performance than any other controller pairing or any other design condition. Even though this driving force approach considered only binary systems, the method has been extended to handle also multicomponent systems by Sanchez-Daza et al. (2003). Figure 8 shows the work-flow for integrated design-control of reaction-separation processes using the graphical design approach. 


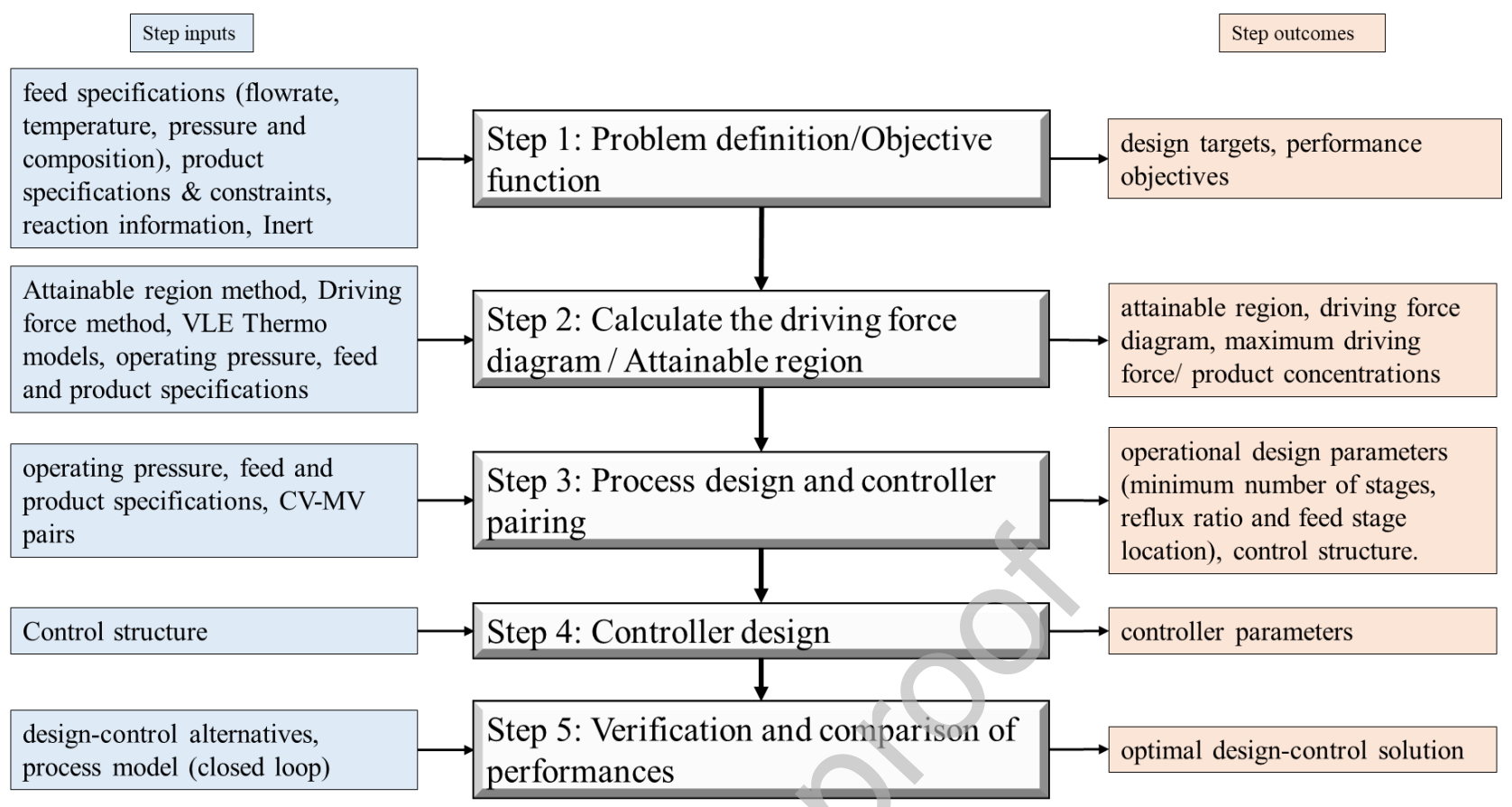

Figure 8: Work-flow for graphical-design based decomposition approach

Step 1: Problem definition: In this step the problem of design and control is defined. The data/information on raw materials, products, catalysts, reaction conversions, and feed conditions (temperature, pressure, and composition) along with product specifications like purity, concentration are defined in this step. Furthermore, the objective function which is to be maximized or minimized from both design and control perspectives is defined in this step. The objective function may be in the form of a weighted multi objective function or a set of process design and control performance metrics which are to be maximized or minimized.

Step 2: Calculation of the driving force diagram /attainable region plots: In this step driving force plot and the attainable region plots are constructed based on the unit operations involved. The maximum points of this plots are used in subsequent steps to generate design parameters. Note that for this step, process design information is not necessary. Only the chemical system (with or without reaction) data is necessary.

Step 3: Process design and controller pairing: The objective of this step is to find the design parameters and best controller pairing for all the available CV's and MV's. From maximum available driving force 
and attainable region, design parameters like number stages, feed stage location, reflux ratio, reactor product concentration, reactor volume etc. are obtained. Optimal control structure is also determined using these methods. For example, by using the attainable region concept for reactors, the selection of the primary controlled variable is fixed at the $y$-axis of the attainable region diagram which is product concentration, since it effects the objective function. That is, at the highest attainable point for the product concentration, it is possible to obtain the corresponding reactant concentration and the temperature at which the reaction needs to be performed (if the kinetic model includes temperature dependence). Note also that the highest product concentration also corresponds to the largest product yield. See also Lopez-Arenas et al. (2019) for driving force-based analysis of reactive and non-reactive systems.

Step 4: Controller design: In this step the controller type and parameters are identified. Here the controller design is performed by following the steps outlined in section 3.3.

Step 5: Verification and comparison of performances: In this step the design-control alternatives found in step 3-4 are verified using different analysis based on closed-loop simulations. The analysis includes performing simulations with rigorous dynamic models for specified controller parameters for disturbance rejection and set point tracking. Furthermore, the control structure is also verified using relative gain analysis for different controller pairings.

\subsubsection{Mathematical programming-based optimization approach}

In this approach the design and control parameters are optimized based on mathematical formulation involving design and controller decisions. The method based on this approach, as shown in Figure 9, has 4 steps.

Step 1: Problem definition: In this step the problem of design-and control is defined. The data/information on raw materials, products, catalysts, reaction conversions, and feed conditions (temperature, pressure, and composition) along with product specifications like purity, concentration are defined in this step. Furthermore, the objective function, which is to be maximized or minimized from both design and control perspectives is defined in this step. The objective function may be in the 
form of a weighted multi objective function or a set of process design and control performance metrics which are to be maximized or minimized.

Step 2: Determination of initial design parameters: In this step driving force plot and the attainable region plots are constructed based on the unit operations involved. The maximum points of these plots are used in to determine the design parameters like number stages, feed stage location, reflux ratio, reactor product concentration, reactor volume. The design parameters estimated using these methods are proved to be very close to optimal operation points.

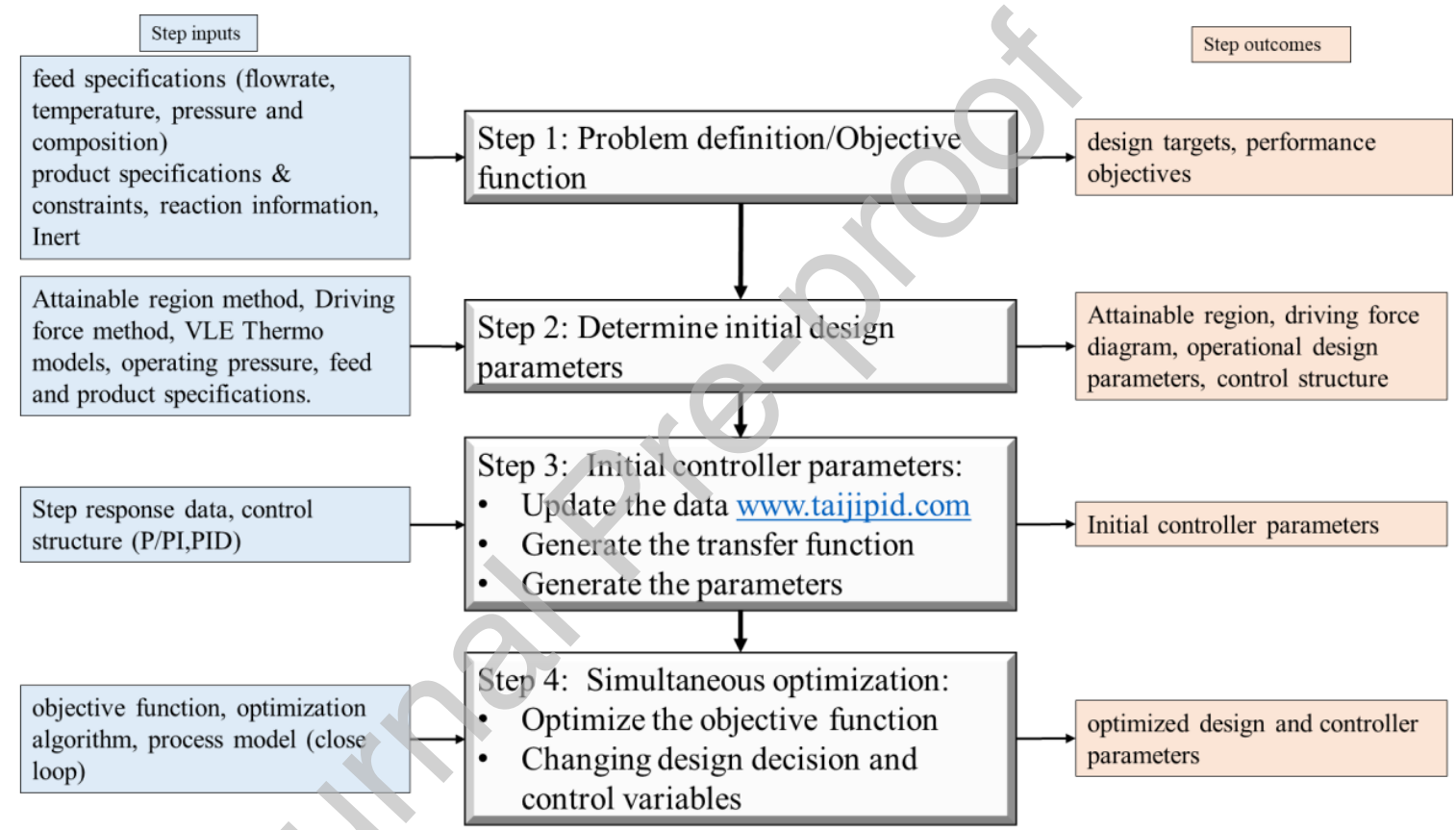

Figure 9: Work-flow for mathematical programming-based optimization approach

Step 3: Initial controller parameters: In this step for a selected controller structure, the controller parameters are determined. First transient response data is generated to obtain the transfer function coefficients and the PID controller parameters.

Step 4: Optimization problem and solution: Here, the objective function includes measures of control performance and process economics. The estimated design parameters (from step 2) and controller 
parameters (from step 3) are used as initial values for the optimization step. The flow-diagram for this optimization step is in Figure 10.

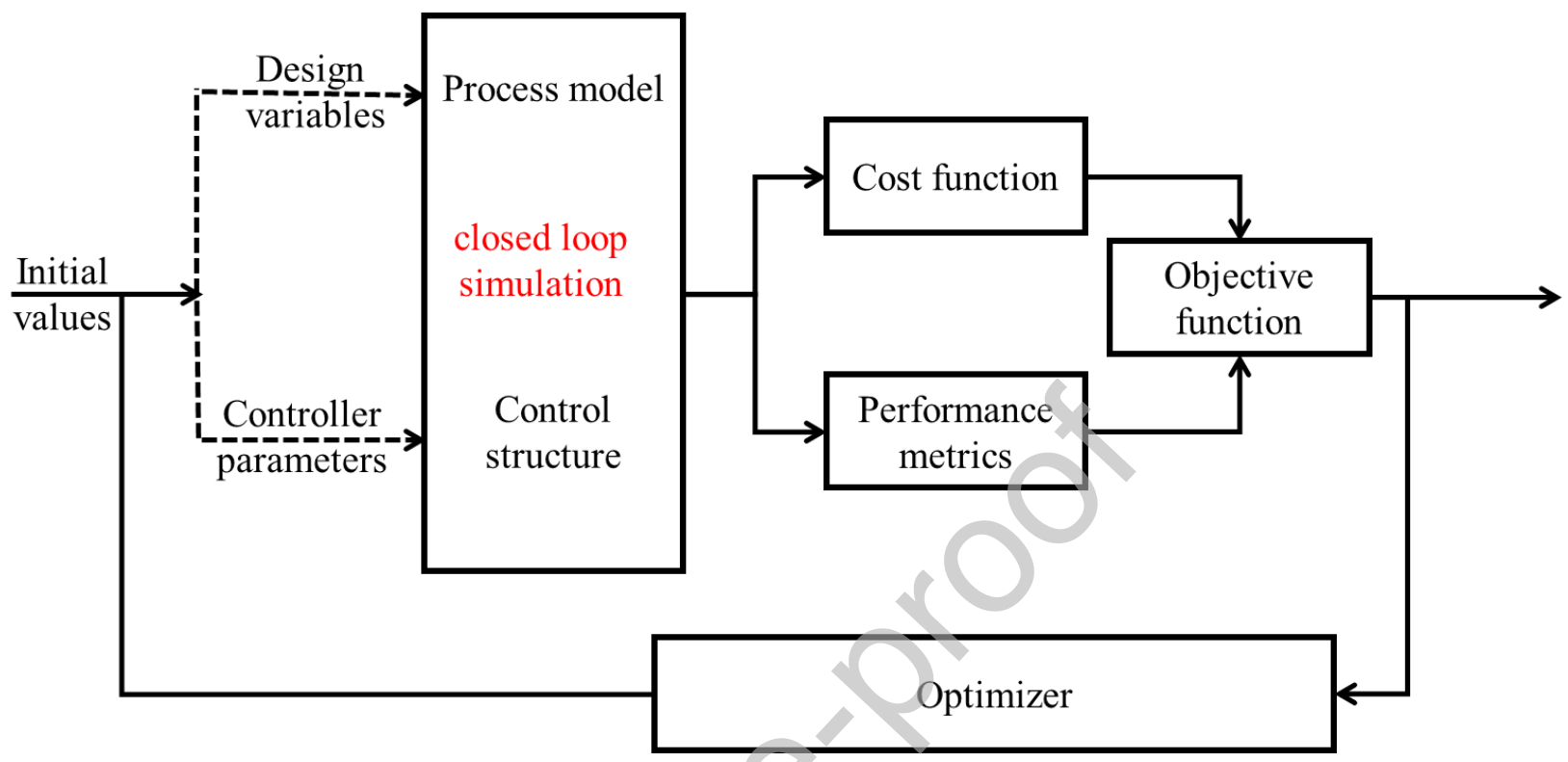

Figure 10: Mathematical programming-based optimization approach for design-control

As shown in Figure 10, in the inner-loop simulations are performed with a selected closed-loop model with the initial estimates of the design and controller parameters. These simulation results are used to calculate the objective function, which consists of different terms: cost function including both the capital and operating cost terms corresponding to the design decision, and performance index for the control decision. The results from the inner-loop (objective function value and the associated terms and variables) is sent to the outer-loop where a suitable solver, for example, the gradient free optimization algorithm (BOBYQA) is employed to obtain new values of design and controller parameters. This iterative calculation continues until convergence of the outer-loop is achieved. It should be noted that the iterative process may not be guaranteed to give the global optimal solution as it may be stuck into a local optimum.

\subsection{Integrated Tools}


In this section a detailed overview of the tool's library of ProCACD is given. This integration of different tools gives access to different components, which are used during the solution of various process control related problems.

\subsubsection{Aspen Dynamics}

ProCACD is integrated with commercial dynamic simulators like Aspen Dynamics (Aspen Technology, 2019c), where the final process and controller design can be verified using the rigorous models. The integration is done through Aspen Dynamics Input language elements by which an input file which is compatible with Aspen Dynamics is generated from ProCACD. Based on these elements a template is developed in ProCACD, which includes compounds data, unit operations data with both design parameters and connections, controller design and other process related specifications. Based on this template an input file is generated which can be directly opened using Aspen Dynamics which converts all the data from the input file to a complete process flowsheet. This integration can be used to generate different results like controller performance, design-control verifications based on dynamic rigorous simulations. Figure 11 shows the integration and work-flow of ProCACD with Aspen Dynamics. 


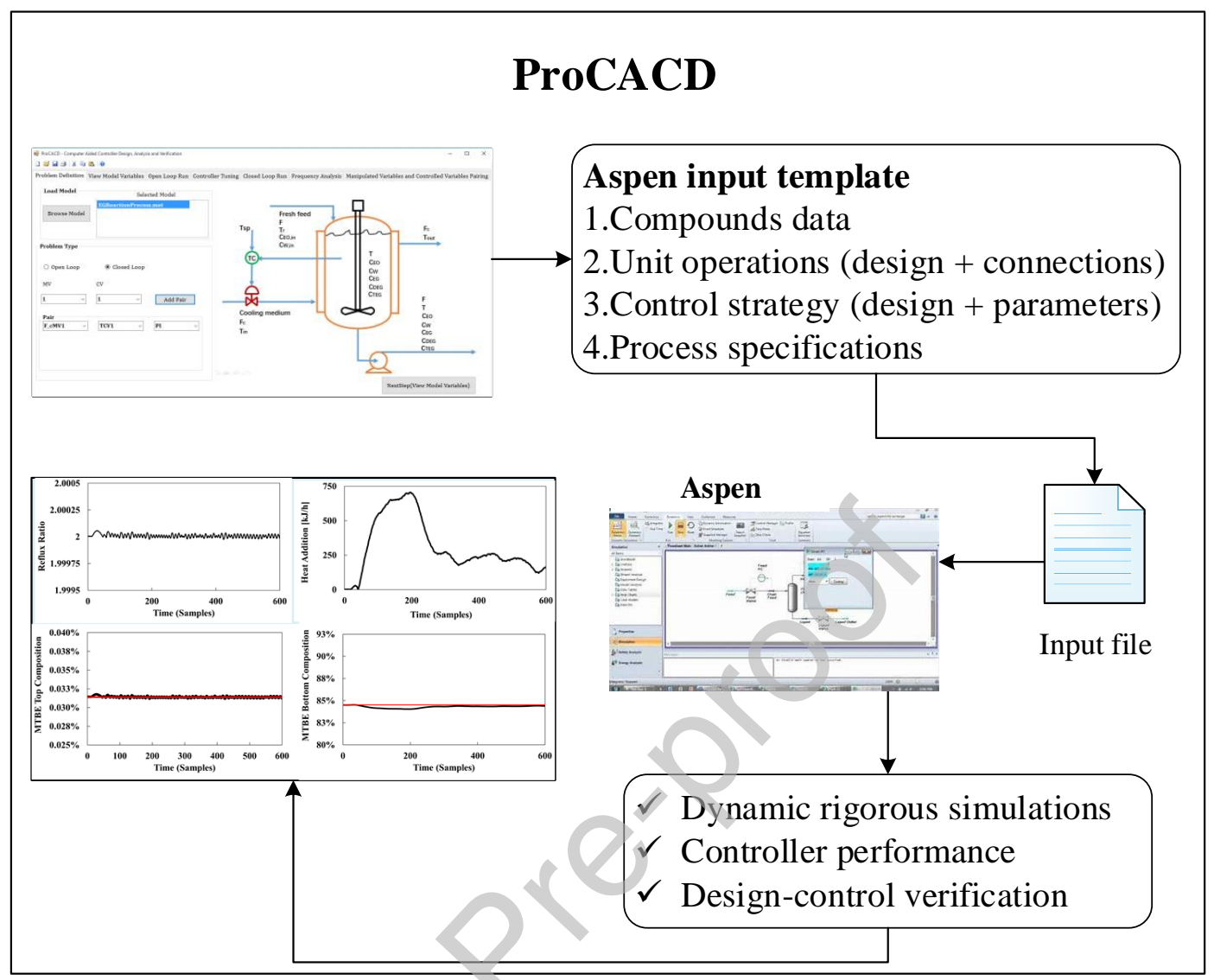

Figure 11: Integration of Aspen and ProCACD

\subsubsection{ICAS-Sim}

ICAS-Sim (Gani et al., 1997) is an in-house tool, which is integrated with ProCACD. Similar to Aspen, ICAS-Sim is also used to run dynamic simulations to verify process and controller design decisions. However, ProCACD can directly send the information to ICAS-Sim without the need for an input file. With the input information, ProCACD invokes the simulator and generates the rigorous flowsheet. Here ICAS-Sim can also be used to carry other analysis like generating driving for diagrams, calculating design parameters for unit operations etc. unlike Aspen Dynamics, where it is used to run closed-loop simulations. Figure 12 shows the integration and work-flow of ProCACD with ICAS-Sim. 


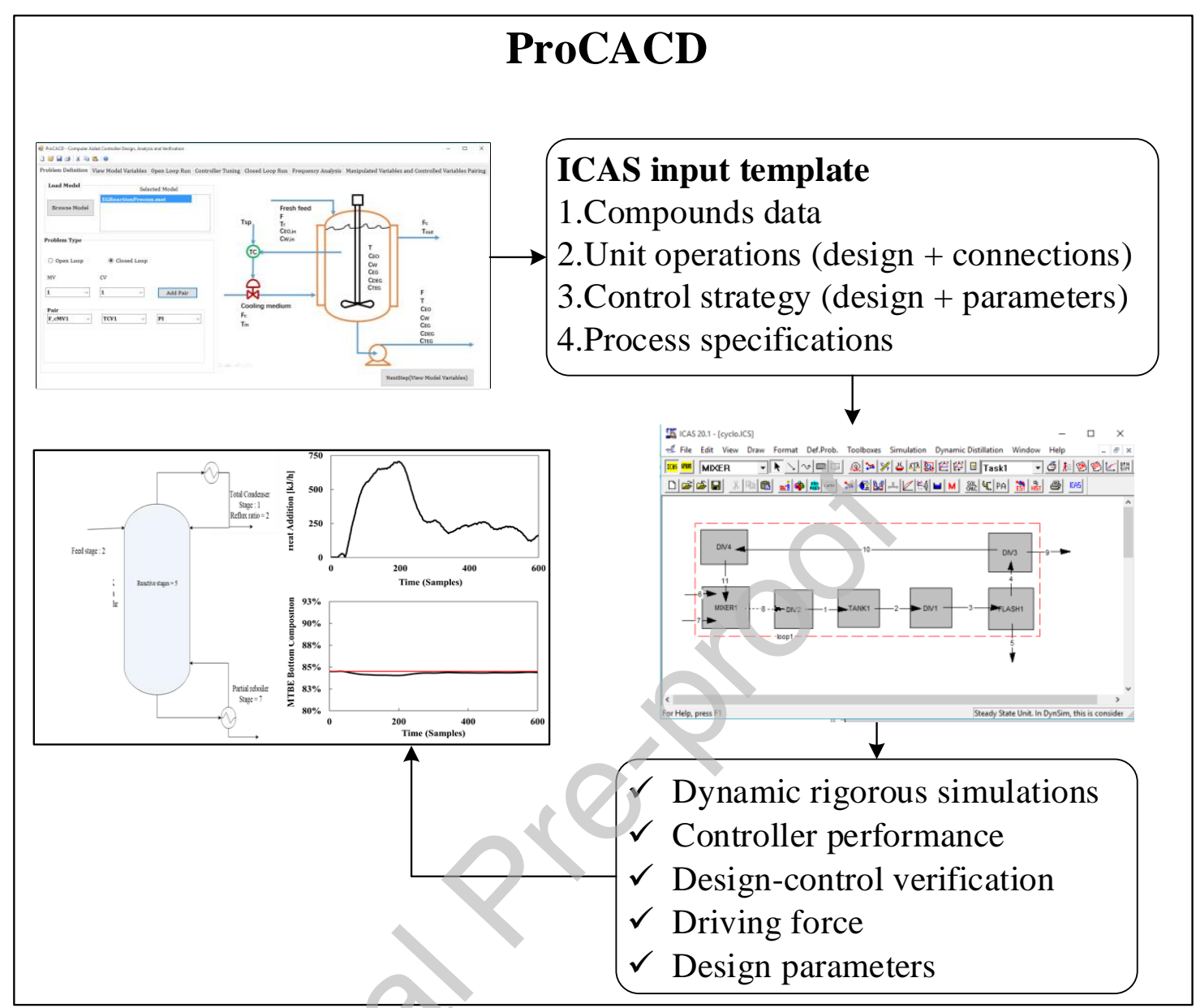

Figure 12: Integration of ICAS-Sim and ProCACD

\subsubsection{Integration of MoT}

ICAS-MoT (Sales-Cruz and Gani, 2003) is an in-house tool, which is used for model construction, analysis and solution. It has built-in solution algorithms and solvers used for solving different types of numerical problems. MoT is integrated with ProCACD such that the mode of dynamic simulation (open-loop/closed-loop) is directly configured in ProCACD. This is achieved through the use of COMObjects based on which loading, manipulation and evaluation of ICAS-MoT models are carried out in ProCACD. In ProCACD, MoT is mainly used for model construction (or external model transfer) and for dynamic simulations. Figure 13 shows the integration work-flow of ProCACD and MoT. 


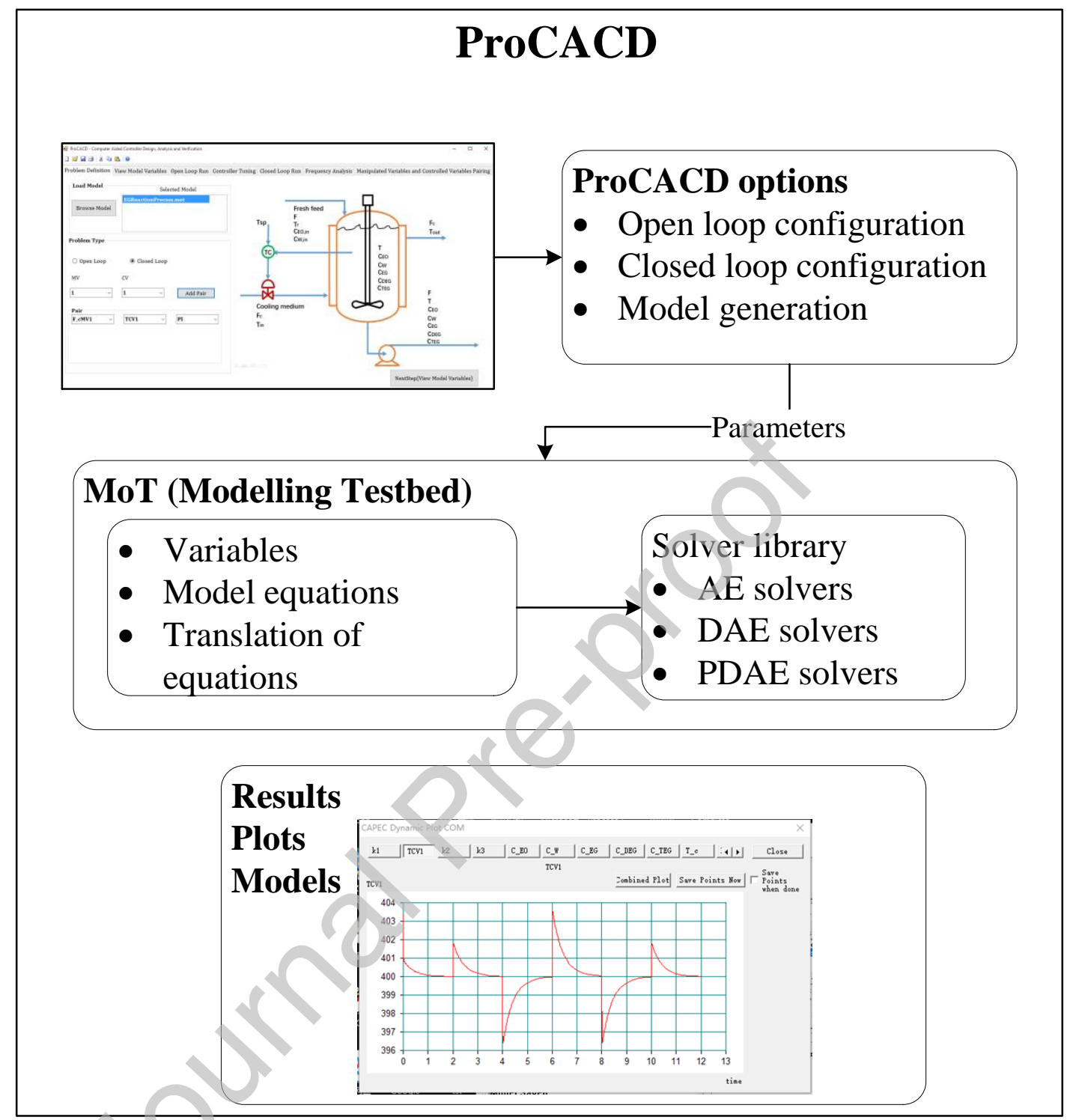

Figure 13: Integration of MoT and ProCACD

\section{Application Examples}

Application examples highlighting selected features of ProCACD are presented in this section. The first two examples highlight the application of the work-flow and associated tools for the solution of typical control related problems. The last two examples highlight the application of work-flow and associated tools for solution of two integrated process design-control problems. The model library contains a large number of process models (single unit operations as well as process flowsheets, see section S1 in 
supplementary materials), any of which, in principle can be retrieved and investigated for control related issues. Also, many more solved case studies can be obtained from the authors.

\subsection{Three-Tank System: Analysis}

To demonstrate the ability of ProCACD to develop (and/or analyze) retrieved process models and perform simulations efficiently and flexibly, a simple conceptual example involving a three-tank system is presented. The analysis is carried out by performing open-loop and closed-loop simulations with the three tank model, using a simple first principle-based model and a generated transfer functionbased model.

\subsubsection{Problem definition}

This example is taken from (Marchetti et al., 2009), which is an interacting three tank system as shown in Figure 14. In this system the three tanks are equipped with three control valves (V1, V2, V3) to control the liquid level in the respective tanks. The system also has automatic two-way valves V12 and V32 which determines the liquid flow direction based on the level difference. Liquid is fed into tanks 1 $\& 3$. Figure 14 shows the closed-loop configuration of the three-tank model and the generic model equations representing this system are given by Equations 6-25. 


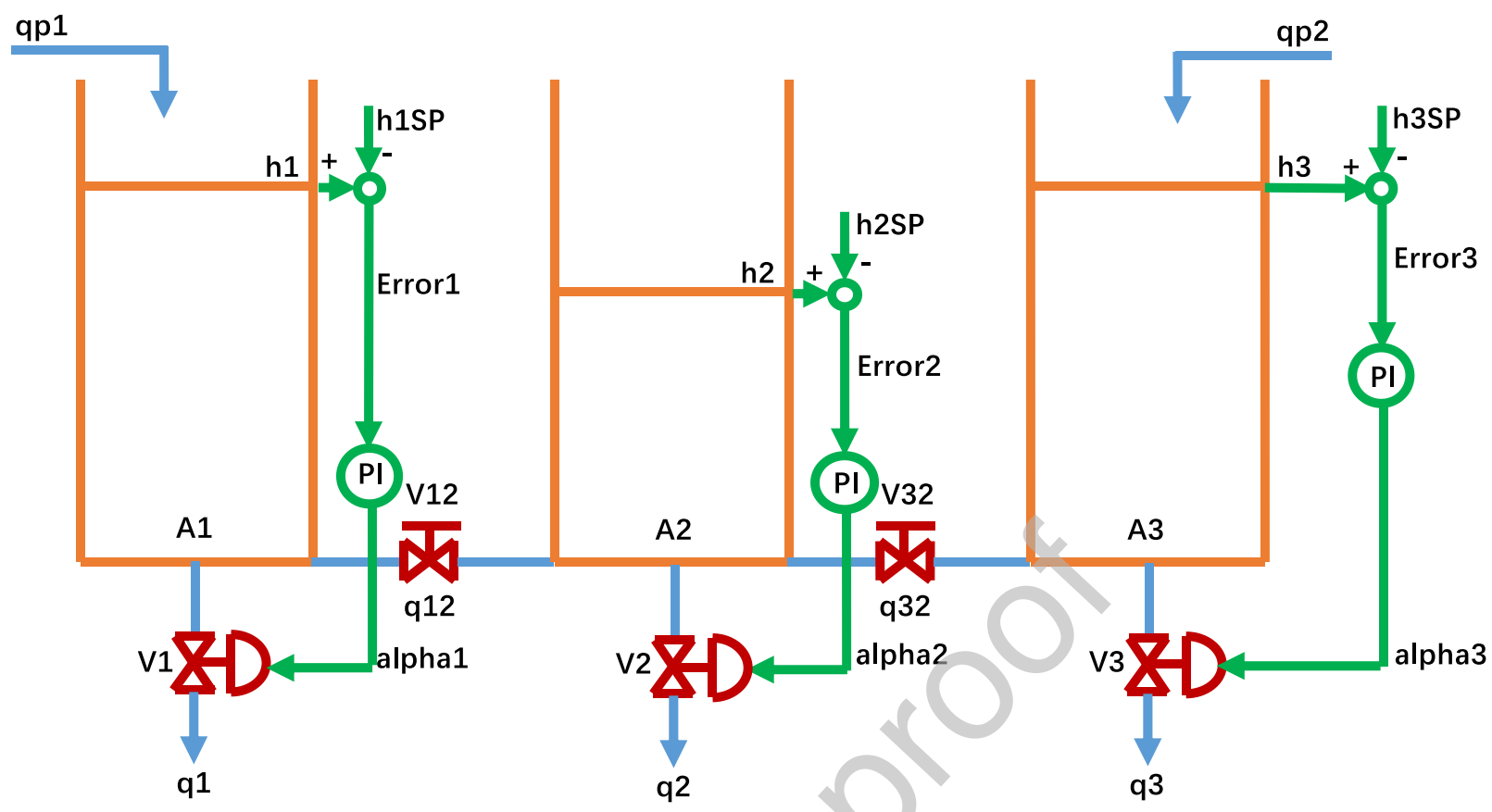

Figure 14: Three-Tank model

\subsubsection{Process model}

Balance equations

$\operatorname{dh} 1 C V 1=\frac{q p 1-q 1-q 12}{A 1}$

$d h 2 C V 2=\frac{q 12+q 32-q 2}{A 2}$

$\operatorname{dh} 3 C V 3=\frac{q p 2-q 3-q 32}{A 3}$

Constitutive equations

$q 1=A 1 * \operatorname{alpha} 1 M V 1 * \sqrt{h 1 C V 1}$

$q 2=A 2 * \operatorname{alpha} 2 M V 2 * \sqrt{h 2 C V 2}$

$q 3=A 3 *$ alpha $3 M V 3 * \sqrt{h 3 C V 3}$ 
$q 12=i f(h 1 C V 1>h 2 C V 2) A 1 *$ alpha $12 * \sqrt{h 1 C V 1-h 2 C V 2}$ else - A2 $*$ alpha $12 *$ $\sqrt{h 2 C V 2-h 1 C V 1}$

$q 32=i f(h 3 C V 3>h 2 C V 2) A 3 *$ alpha $32 * \sqrt{h 3 C V 3-h 2 C V 2}$ else - A2 *alpha32 *

$\sqrt{h 2 C V 2-h 3 C V 3}$

Control (conditional) equations

Error $1=h 1 C V 1-h 1 C V 1 S P$

Error $2=h 2 C V 2-h 2 C V 2 S P$

Error $3=h 3 C V 3-h 3 C V 3 S P$

$\mathrm{d} A E 1=$ Error 1

$\mathrm{d} A E 2=$ Error 2

$\mathrm{d} A E 3=$ Error 3

alpha1MV1 $=$ alpha1MV1 $*$ ol1 $+((P 1 *$ Error $1+$ alpha1MV1Star $) * \operatorname{clp} 1+(P 1 *$ Error $1+I 1 * A E 1+$ alpha1MV1Star $) * \operatorname{clpi} 1+(P 1 *$ Error $1+I 1 * A E 1+D 1 * \operatorname{dh} 1 C V 1+$ alpha1MV1Star $) *$ clpid 1$) *$ Pair1_1 + $((P 1 *$ Error $2+$ alpha1MV1Star $) * \operatorname{clp} 1+(P 1 *$ Error $2+I 1 * A E 2+$ alpha $1 M V 1 S t a r) * \operatorname{clpi} 1+(P 1 *$ Error $2+I 1 * A E 2+D 1 *$ dh $2 C V 2+$ alpha1MV1Star $) *$ clpid1 $) *$ Pair1_2 + $((P 1 *$ Error $3+$ alpha1MV1Star $) * \operatorname{clp} 1+(P 1 *$ Error $3+I 1 * A E 3+$ alpha $1 M V 1$ Star $) * \operatorname{clpi} 1+(P 1 *$ Error $3+I 1 * A E 3+D 1 *$ dh $3 C V 3+$ alpha1MV1Star) * clpid1) * Pair1_3

alpha $2 M V 2=$ alpha2MV2 $*$ ol $2+((P 2 *$ Error $1+$ alpha $2 M V 2 S t a r) * \operatorname{clp} 2+(P 2 *$ Error $1+I 2 * A E 1+$ alpha2MV2Star $) *$ clpi $2+(P 2 *$ Error $1+I 2 * A E 1+D 2 *$ dh $1 C V 1+$ alpha2MV2Star $) *$ clpid2) $*$ Pair2_1 + ((P2 * Error $2+$ alpha2MV2Star $) * \operatorname{clp} 2+(P 2 *$ Error $2+I 2 * A E 2+$ alpha $2 M V 2 S t a r) * \operatorname{clpi} 2+(P 2 *$ Error $2+I 2 * A E 2+D 2 *$ dh $2 C V 2+$ alpha2MV2Star $) *$ clpid2 $) *$ Pair2_2 $+((P 2 *$ Error $3+$ alpha2MV2Star $) * \operatorname{clp} 2+(P 2 *$ 
Error $3+I 2 * A E 3+$ alpha $2 M V 2 S t a r) * \operatorname{clpi} 2+(P 2 *$ Error $3+I 2 * A E 3+D 2 *$ dh $3 C V 3+$ alpha2MV2Star) $*$ clpid2) *Pair2_3

alpha3MV3 $=$ alpha3MV3 $*$ ol3 $+((P 3 *$ Error $1+$ alpha3MV3Star $) * \operatorname{clp} 3+(P 3 *$ Error $1+I 3 * A E 1+$ alpha3MV3Star $) *$ clpi $3+(P 3 *$ Error $1+I 3 * A E 1+D 3 *$ dh $1 C V 1+$ alpha3MV3Star $) *$ clpid3 $) *$ Pair3_1 + ((P3 * Error $2+$ alpha3MV3Star $) * \operatorname{clp} 3+(P 3 *$ Error $2+I 3 * A E 2+$ alpha3MV3Star $) *$ clpi3 $+(P 3 *$ Error $2+I 3 * A E 2+D 3 *$ dh $2 C V 2+$ alpha3MV3Star $) *$ clpid3 $) *$ Pair3_2 $+((P 3 *$ Error $3+$ alpha3MV3Star $) * \operatorname{clp} 3+(P 3 *$ Error $3+I 3 * A E 3+$ alpha3MV3Star $) *$ clpi $3+(P 3 *$ Error $3+I 3 * A E 3+D 3 *$ dh $3 C V 3+$ alpha3MV3Star) * clpid3) *Pair3_3

Equality and/or inequality constraints

$0 \leq$ alpha1MV1 $\leq 1$

$0 \leq$ alpha2MV2 $\leq 1$

$0 \leq$ alpha3MV3 $\leq 1$

In the above equations, $h 1 C V 1, h 2 C V 2$ and $h 3 C V 3$ (controlled variables) are the levels of the liquid in three tanks. $A_{1}, A_{2}$ and $A_{3}$ are the cross-sectional areas of three tanks. $q p 1$ and $q p 2$ are the inlet flowrates of tanks 1 and 3 on both sides. $q 1, q 2$ and $q 3$ are the outlet flowrates of three tanks. $q 12$ and $q 32$ are the flowrates between tanks. alpha1MV1, alpha2MV2 and alpha3MV3 (manipulated variables) are bottom valve coefficients of three tanks. alpha12 and alpha32 are valve coefficients between tanks. h1CV1SP, $h 2 C V 2 S P$ and $h 3 C V 3 S P$ are the setpoint levels in three tanks. Error1, Error2 and Error3 are the errors between controlled variables and setpoints. AE1, AE2 and AE3 are the integrals of Error1, Error2 and Error3. P1, I1, D1, P2, I2, D2, P3, I3 and D3 are controller parameters and alpha1MV1Star, alpha2MV2Star and alpha3MV3Star are the steady state coefficients of the bottom valves. ol1, ol2, ol3, clp1, clp2, clp3, clpi1, clpi2, clpi3, clpid1, clpid2, clpid3, Pair1_1, Pair1_2, Pair1_3, Pair2_1, Pair2_2, Pair2_3, Pair3_1, Pair3_2 and Pair3_3 are binary variables to configure the structure of the control model. However, in this example the since the control structure is predetermined only Pair1_1, Pair2_2 and Pair3_3 are enabled. Table 2 gives the binary integer values for these parameters 
corresponding to different controller models, which ProCACD employs to upload and use in different closed-loop simulation models. We use loop alpha1MV1- h1CV1 for example. Table 3 gives the specified values for the three-tank model design variables.

Table 2: Binary values for different model configurations for loop alpha1MV1- h1CV1

\begin{tabular}{|c|c|c|c|c|c|}
\hline & \multicolumn{4}{|c|}{ Binary variables } & \multirow[t]{2}{*}{ Model application } \\
\hline ol1 & clp1 & clpi1 & clpid1 & Pair1_1 & \\
\hline 1 & 0 & 0 & 0 & 0 & open-loop \\
\hline 0 & 1 & 0 & 0 & 1 & closed-loop with P \\
\hline 0 & 0 & 1 & 0 & 1 & closed-loop with PI \\
\hline 0 & 0 & 0 & 1 & & closed-loop with PID \\
\hline
\end{tabular}

Table 3: Specified values in the three-tank model

\begin{tabular}{|l|l|c|}
\hline Inlet flowrate of tank 1 & $q p 1\left(\mathrm{~cm}^{3} / \mathrm{min}\right)$ & 79.32 \\
\hline Inlet flowrate of tank 3 & $q p 2\left(\mathrm{~cm}^{3} / \mathrm{min}\right)$ & 92.75 \\
\hline Cross-sectional area of tank 1 & $A 1\left(\mathrm{~cm}^{2}\right)$ & 100 \\
\hline Cross-sectional area of tank 2 & $A 2\left(\mathrm{~cm}^{2}\right)$ & 154 \\
\hline Cross-sectional area of tank 3 & $A 3\left(\mathrm{~cm}^{2}\right)$ & 200 \\
\hline Coefficient of valve between tank 1,2 & alpha12 & 0.0381 \\
\hline Coefficient of valve between tank 3,2 & alpha32 & 0.0285 \\
\hline
\end{tabular}

\subsubsection{Open-loop simulation}

The open-loop simulation is conducted by setting "oll", "ol2" and "ol3" as 1 and all other binary parameters to 0 in the control model. The system model is analyzed to check the stability of the openloop process response for step changes on bottom valve coefficients (alpha1MV1, alpha2MV2 and alpha3MV3). The bottom valve coefficients are increased by $100 \%$ from 0.1203 to 0.2406 , from 0.0613 to 0.1226 , from 0.1141 to 0.2282 , respectively, and the open-loop transient responses are generated. 
The detailed plots of the open-loop responses are shown in section S2 (supplementary material). From the simulated responses, it is seen that the tanks' level ( $h 1 C V 1, h 2 C V 2$ and $h 3 C V 3)$ always go to a new steady state for each step change. Also, it can be observed that the process has reached a new steady quickly for these step changes.

The open-loop stability can also be analyzed by ProCACD through transfer function model. ProCACD use system identification to identify the transfer function model of each loop based on open-loop simulation data. Thus, ProCACD can get the open-loop characteristic equations and use Routh stability criterion to analyze the stability of the open-loop system. For example, loop alpha1MV1- $h 1 C V 1$ characteristic equation is given by Equation 26. According to Routh stability criterion, the loop is stable at this operating condition.

$33.58 s+1=0$

\subsubsection{Closed-loop simulation}

The closed-loop simulations are performed to analyze the process with respect to performance of a feedback controller. Here PI controllers are used to regulate the coefficients of the bottom valves (alpha1MV1, alpha2MV2 and alpha3MV3) such that the tank levels ( $h 1 C V 1, h 2 C V 2$ and $h 3 C V 3)$ can be maintained at the set points. The controller parameters used in this example are calculated using ProCACD (controller design option) as given in section S3 (supplementary material).

In order to analyze the process for this feedback controller a set point change on level of tank 2 ( $h 2 C V 2 \mathrm{SP}$ ) is given. The level is first increased from $8.7 \mathrm{~cm}$ to $10 \mathrm{~cm}$ and then decreased to $8 \mathrm{~cm}$ and finally brought back to $8.7 \mathrm{~cm}$. Figure $15 \mathrm{a}$ shows the tracking of the tank level for the changed set point values. Figure $15 \mathrm{~b}$ shows the corresponding change in the manipulated variable (tank 2 bottom valve coefficient) in order to track the different set points. 

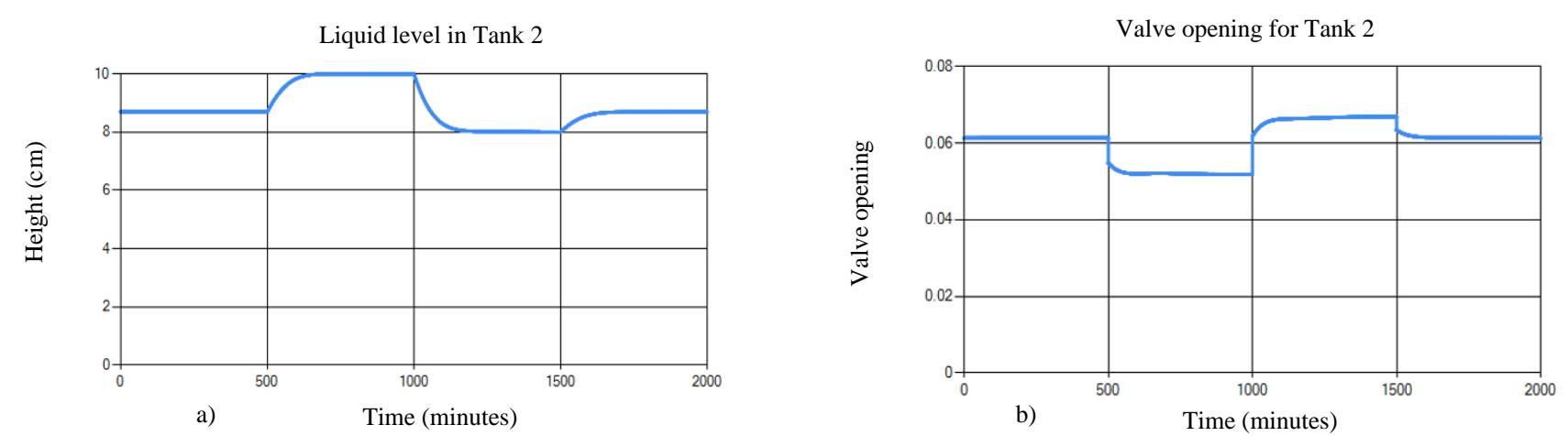

Figure 15: Set point tracking for three-tank model using PI controllers a) level b) valve coefficient

The process is also analyzed to evaluate the effect of disturbances on the process. This is achieved in ProCACD by giving a step change to the inlet flowrate of tank 1 (changed from $79.32 \mathrm{~cm}^{3} / \mathrm{min}$ to $100 \mathrm{~cm}^{3} / \mathrm{min}$, then to $79.32 \mathrm{~cm}^{3} / \mathrm{min}$, then back to $100 \mathrm{~cm}^{3} / \mathrm{min}$ ). Figure 16 a shows the effect of disturbances and controller actions on level of tank 1, while Figure $16 \mathrm{~b}$ shows the responses of the controller where the tank 1 valve coefficient is quickly manipulated to maintain the set point level of $27.32 \mathrm{~cm}$.

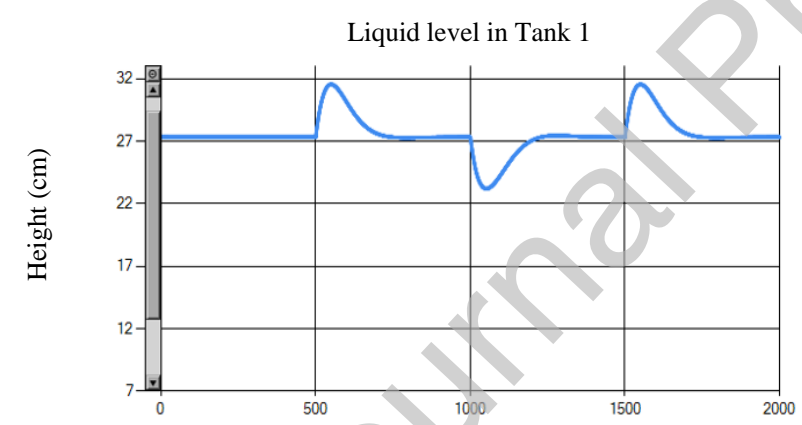

a)

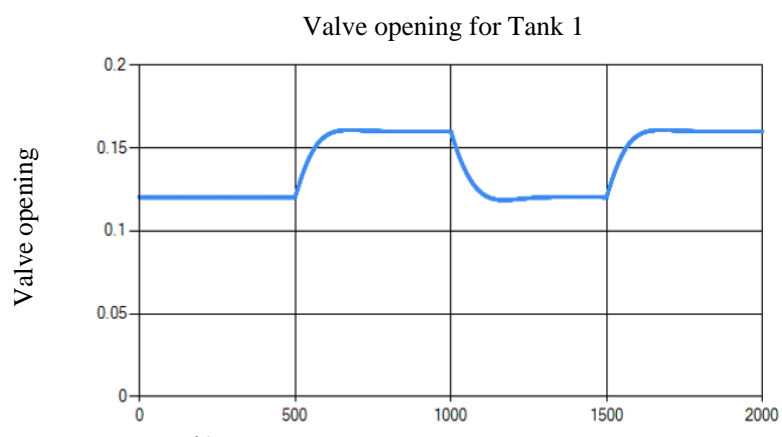

b)

Figure 16: Disturbance rejection for three-tank model using PI controllers a) level b) valve coefficient

For this case study, it is clear from the analysis results that the PI based feedback controller shows good disturbance rejection and set point tracking. Also, from the open-loop analysis it was found that the model was able to capture the step changes on valve coefficients and their effects on the tank's level. The objective of the case study, however, is to illustrate that this standard control related analysis, system identification can be easily handled by ProCACD.

\subsection{Optimal Controller Design}


The controller design option developed in ProCACD, is highlighted where the controller is designed for the system introduced in 4.1. The work-flow described in section 3.3 is applied for this example. The model for this process is available in ProCACD and retrieved from the model library. The generic model equations are given by Equations 6-25 (see section 4.1)

\subsubsection{Problem definition}

The objective of this example is to determine an optimal PID controller design to maintain the level of the liquid in each of the three tanks subjected to disturbances in feed or set point. In the first step optimal pairing of control and manipulated variable is identified and in the second step the controller parameters are tuned. Note that each tank has different dimensions and therefore different set-points for level control and flow manipulation.

\subsubsection{Controller pairing}

There is a total of 3 controlled variables and 3 manipulated variables in this process. The controlled variables are $h 1 C V 1, h 2 C V 2$ and $h 3 C V 3$, which are heights of liquid levels in the three tanks. The manipulated variables are alpha1MV1, alpha2MV2and alpha3MV3, which represent the valve openings, respectively for the exit flows.

Step 1 Step response data collection

Open-loop dynamic simulation is performed, and step response data is generated for each of the manipulated variables. Section S2 (supplementary material) presents the system response for step change on alpha1MV1 (from 0.1203 to 0.2406 ) and corresponding effect on $h 1 C V 1, h 2 C V 2$ and $h 3 C V 3$. Similarly, a step change is given on alpha2MV2 and alpha3MV3, and corresponding response data is generated using ProCACD.

Step 2: Generation of transfer function

ProCACD transfers the response data to Taiji-PID website to estimate the transfer function coefficients. Section S3 (supplementary material) shows the coefficients of the transfer functions for all three loops.

Step 3: Generation of relative gain array

Based on the transfer function information generated in step 2, relative gain array is estimated. From the relative gain array, the best pairing is identified as h1CV1/ alpha1MV1, h2CV2/ alpha2MV2, 
$h 3 C V 3 /$ alpha3MV3 as the value for these pairs in the relative gain array is $1.03,1.14$ and 1.12 respectively; the other values are quite close to zeros or negative. The results are quite straightforward and agree with the experience of engineers. Based on the identified optimal pairing the control structure of this process is shown in Figure 14.

\subsubsection{Controller tuning}

For this example, the on-line method linked to ProCACD is used to tune the PI controller parameters. Section S3 (supplementary material) shows the ProCACD step with the tuned controller parameters for all the three loops.

\subsubsection{Verification}

The controller design is verified through dynamic simulations involving set-point tracking and disturbance rejection. The set-point tracking and disturbance rejection simulation results are presented in 4.14. The closed-loop simulation results confirm that the controllers perform well both in disturbance rejection and set point tracking using the tuned controller parameters.

The controller design is also verified through model uncertainty analysis and stability analysis. For model uncertainty analysis, a $10 \%$ change of the cross-sectional area of tank 1 is applied $\left(A 1=90 \mathrm{~cm}^{2}\right)$. And we do verification of set-point tracking under the model uncertainty. A set point change (8.7 to 10, 10 to 8 and back to 8.7 ) is made on $h 2 C V 2$. Figure 17 a shows the tracking of the set-point on $h 2 C V 2$ and Figure $17 \mathrm{~b}$ shows the change in valve opening to reach the new set point. The closed-loop simulation results confirm that the controllers perform well under model uncertainty using the tuned controller parameters. 

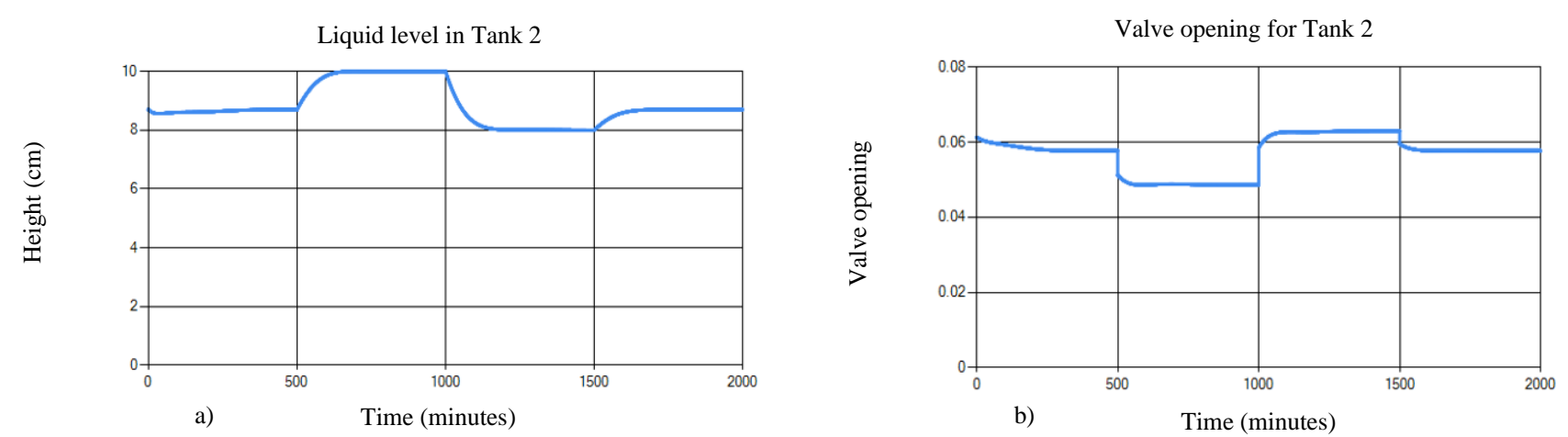

Figure 17: Disturbance rejection for 3 tanks in series model. a) Liquid level b) Valve opening

ProCACD also provides stability analysis for closed loop. ProCACD calculates the closed-loop characteristic equations. For example, loop 1 representing alpha $1 M V 1-h 1 C V 1$ has the characteristic equation given by Equation 27. It is shown that all the three control loops are stable under the operating condition.

$33.58 s^{2}+1.43 s+0.0124=0$

\subsection{Integrated Design and Control - I}

This application highlights the use of the integrated process design and control option in ProCACD (see section 3.5.2) through a reactor process to produce Ethylene Glycol (EG). The reactor is a CSTR and the corresponding model and associated parameters are taken from Hamid et al. (2010).

The production of EG involves isothermal, irreversible multi-step consecutive-parallel liquid phase reactions, given by,

$$
\begin{gathered}
\mathrm{C}_{2} \mathrm{H}_{4} \mathrm{O}+\mathrm{H}_{2} \mathrm{O} \rightarrow \mathrm{C}_{2} \mathrm{H}_{6} \mathrm{O}_{2} \\
\mathrm{C}_{2} \mathrm{H}_{6} \mathrm{O}_{2}+\mathrm{C}_{2} \mathrm{H}_{4} \mathrm{O} \rightarrow \mathrm{C}_{4} \mathrm{H}_{10} \mathrm{O}_{3} \\
\mathrm{C}_{4} \mathrm{H}_{10} \mathrm{O}_{3}+\mathrm{C}_{2} \mathrm{H}_{4} \mathrm{O} \rightarrow \mathrm{C}_{6} \mathrm{H}_{14} \mathrm{O}_{4}
\end{gathered}
$$

As shown in equations 28-30, EO (ethylene oxide) and $\mathrm{W}$ (water) react to produce EG. In the side reactions EG reacts with EO to produce diethylene glycol (DEG), and DEG reacts with the remaining EO to produce triethylene glycol (TEG). The optimization approach is employed to design (reactor 
volume) and control (reactor temperature). Figure 18 shows the schematic representation of the reaction process. Model equations representing the generic model for production of ethylene glycol using a CSTR are given in section S4 of supplementary material. The steps involved in solving this case study are highlighted in Figure 9 (see section 3.5.2).

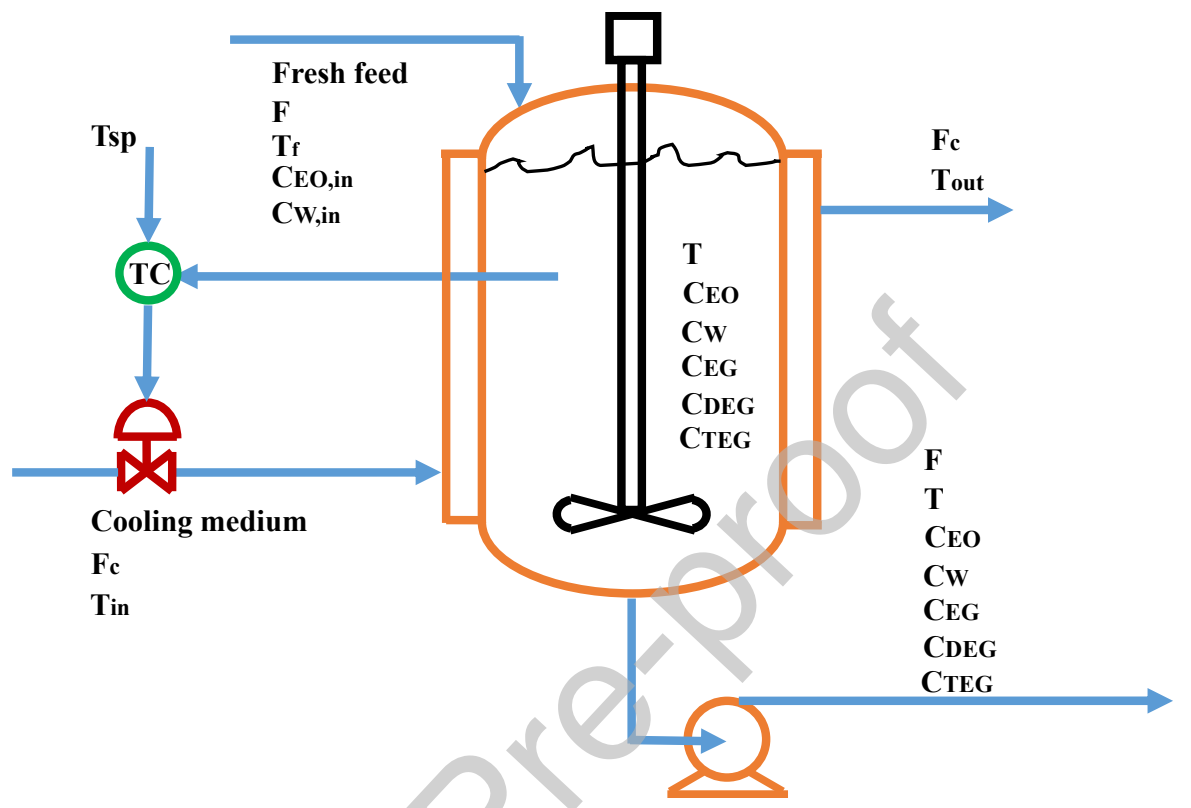

Figure 18: CSTR for production of Ethylene Glycol.

Step 1: Problem definition/Objective

As shown in Figure 10, an inner-and-outer loop calculation is used. In the outer loop, an optimization is conducted. The goal is to find the optimal design and controller structure such that the objective function defined in Equation 31 has a minimum value. The objective function consists of 4 terms, including the control performance index described using ITSE (Integral Time Squared Error) term, the capital cost relating to the size of the reactor $(V R)$, the operational cost relating to the consumption of the utility $\left(F_{c}\right)$, and operational profit relating to the product concentration $\left(C_{E G}\right)$. The optimized variables for the integrated design include the controller parameters (PI controller in this specific case) and the design parameter ( $V R$ in this case). In the inner loop, all the related variables are subject to the process model with closed-loop control. The ITSE term is calculated based on the dynamic simulation results of the controlled variable for specific process and control designs. To maintain a good control performance, an upper bound for the ITSE term is set. Theoretically, this term should be as small as possible, but greater than zero. Note that the real plant is different from simulation results, which do not 
consider many practical aspects like the delay due to the flow in pipes. Therefore, for consistency with practical problems, a lower bound for the ITSE term is also set to make the control system robust. The product specification includes a minimum concentration of $0.16 \mathrm{kmol} / \mathrm{m} 3$ for $\mathrm{EG}$ coming out of the reactor. Similarly, the bounds for volume of the reactor are set to be $3 \leq V R(\mathrm{~m} 3) \leq 30$ based on Sinnot (2005). Section S4 of supplementary material gives the inlet feed conditions (concentration, temperature and other model parameters) used in the process model.

$\min _{P, I, V R}$ obj $=w 1 * I T S E+w 2 * V R+w 3 * \frac{1}{C_{E G}}+w 4 * F_{C}$

s.t.

$\left[T, F_{C}, C_{E G}\right]=f\left(P, I, V_{R}\right)$

ITSE $=\int_{0}^{\tau}\left|T_{S P}-T\right| d t$

$3 \leq$ ITSE $\leq 5$

$3 \leq V_{R} \leq 30$

$0 \leq P \leq 1000$

$0 \leq I \leq 1000$

The detailed process model and related equations used as constraints are given in section S4 of supplementary material.

Step 2: Determine initial design parameters

Hamid et al. (2010) proposed a volume (design parameter) of $11.78 \mathrm{~m} 3$ for the CSTR, based on the attainable region. That is, the maximum concentration of the EG is obtained at the highest value of the attainable region for estimated volume of the reactor. Using this value as an initial estimate, the integrated design and control algorithm will determine the optimal value.

Step 3: Determine the initial controller parameters

Based on the process model with estimated design, the controller design algorithm (section 3.3) is employed to obtain an initial estimate of the controller parameters. Note that in this example, the PI 
controller type is pre-selected. With the PID tuning functions, the optimal PI parameters are 0.413 and 65.9326, respectively, as also presented in Table 4. Equation 38 gives the transfer function.

$y(s)=\frac{-0.001 s-0.086}{0.001 s^{2}+0.015 s+1}$

\section{Step 4: Optimization}

Using the initial design and control parameters determined in steps 2 and 3, the mathematical programming problem formulated in Equation 39 is optimized to minimize the overall objective function. Since the range and units are different for each of the terms in the objective function, normalized values are used for the weights in the objective function as follows.

$o b j=I T S E+\frac{V R}{30}+\frac{0.1667}{C_{E G}}+\frac{\mathrm{F}_{\mathrm{c}}}{520.231}$

As mentioned above, a two-tier solution strategy is used in the optimization step. As it is difficult to obtain the gradient information for the outer loop optimization, BOBYQA (Powell, 2009), a gradient free optimization algorithm, is employed with penalties for the constraints. After the outer loop iteratively gives the settings of the process and controller parameters in each iteration, the inner loop is activated for dynamic simulation with closed-loop control. Table 4 gives the optimization settings and results. It is observed that, with the initial settings obtained from steps 2 and 3, the objective function of the integrated design is quite large at the beginning; it is reduced from a value of 167 to 5.3098 through optimization. Figure 19 illustrates the iterative results of the objective function value, from which we can see that convergence is obtained after 171 iterations. Here the initial settings represent the results from sequential approach where the reactor is designed first using the attainable region method and followed by controller design. Using this sequential approach, reactor volume was fixed at $12 \mathrm{~m}^{3}$ and the controller response has an ITSE value corresponding to 6.259. Finally, the objective function calculated for the sequential approach was 166.9. However, the results from the simultaneous design and control show less reactor volume $\left(8.168 \mathrm{~m}^{3}\right)$ with an final objective function value of 5.3098 .

Table 4: Optimization settings and results for EG reaction process

\begin{tabular}{|l|c|c|c|c|c|c|c|}
\hline & $P$ & $I$ & $V R$ & $C_{E G}$ & $F_{c}$ & $I T S E$ & $O b j$ \\
\hline Upper bound & 1000 & 1000 & 30 & - & - & 5 & - \\
\hline
\end{tabular}




\begin{tabular}{|l|c|c|c|c|c|c|c|}
\hline Lower bound & 0 & 0 & 3 & 0.160 & - & 3 & - \\
\hline Initial guesses & 0.413 & 65.932 & 12 & 0.162 & 520.2 & 6.259 & 166.9 \\
\hline Optimal results & 54.735 & 138.04 & 8.168 & 0.1647 & 533.498 & 3 & 5.3098 \\
\hline
\end{tabular}

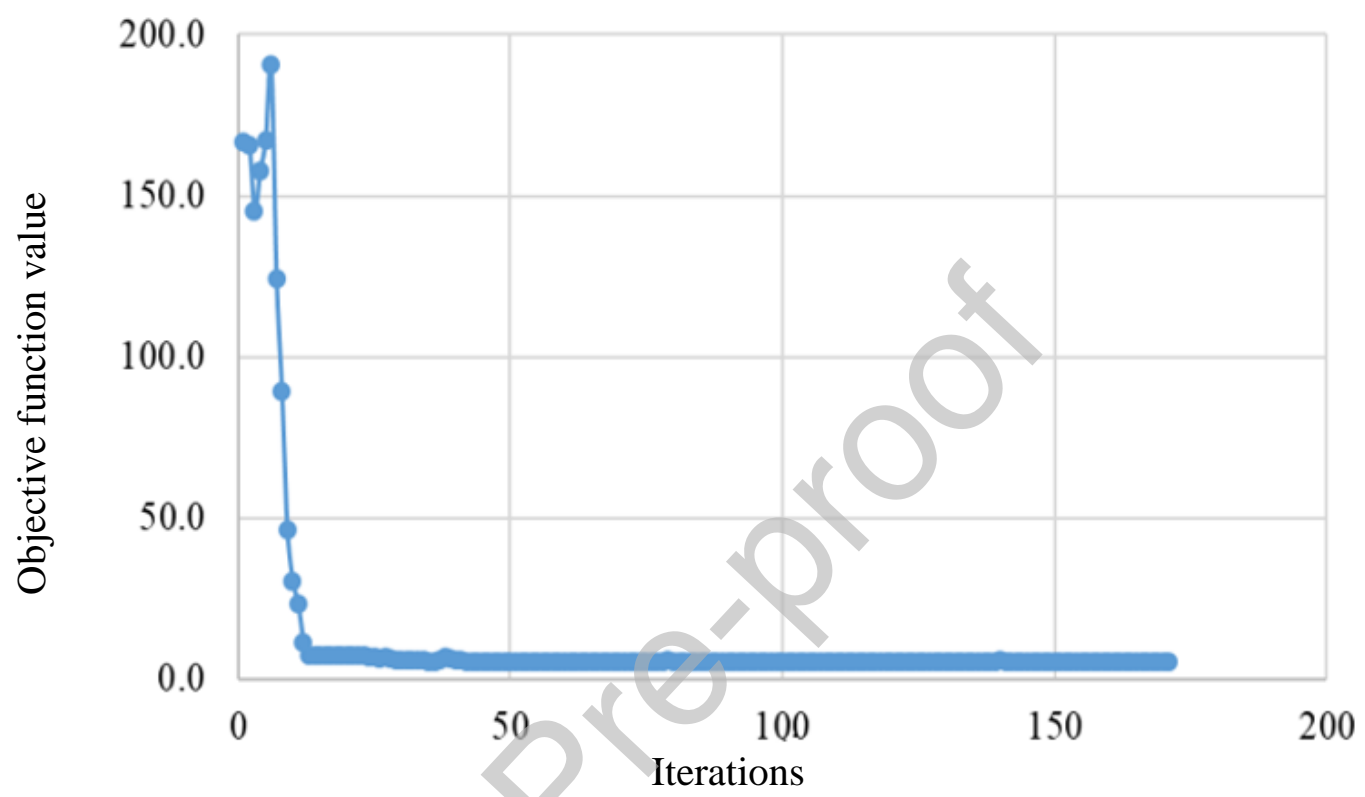

Figure 19: Progress of outer-loop to converged solution

\section{Step 5: Verification}

The optimized design and control parameters are updated with the closed-loop model and a closed-loop dynamic simulation is performed through ProCACD through MoT. Figure 20 shows the tracking of set point (Temperature of reactor, $\mathrm{T})$ for a step disturbance in the feed temperature $\left(500 \pm 10{ }^{\circ} \mathrm{C}\right)$. The blue curve represents the results under the initial parameter settings obtained from steps $2 \& 3$; whereas the yellow curve represents the results under the optimized parameter settings from the final integrated design results. It can be noted that the response with the integrated design-control parameters gives obviously better performance than the initial parameters. 


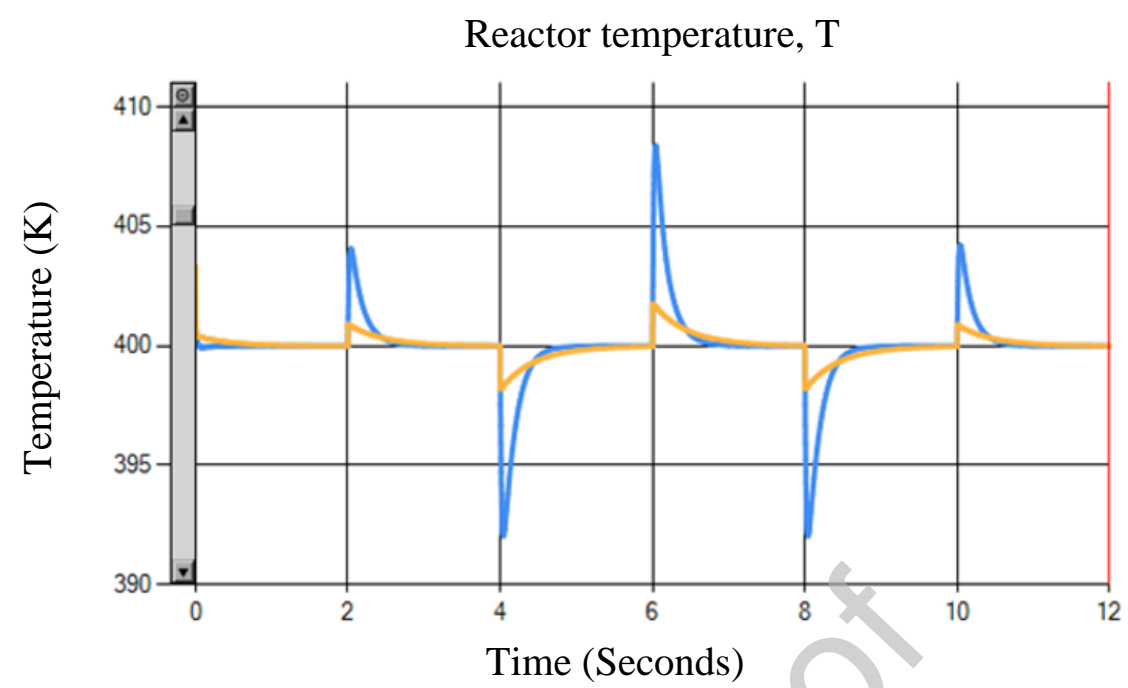

Figure 20: Comparison of disturbance rejection (Blue: initial values, Yellow: Optimized values)

Figure 21 shows the tracking of the set point change of the reactor temperature $\left(400 \pm 1{ }^{\circ} \mathrm{C}\right)$. The blue curve represents the results under the initial parameter settings obtained from steps $2 \& 3$; whereas the yellow curve represents the results under the optimized parameter settings from the final integrated design results. It can be noted that the response with the integrated design-control parameters gives obviously better performance than the initial parameter setting.

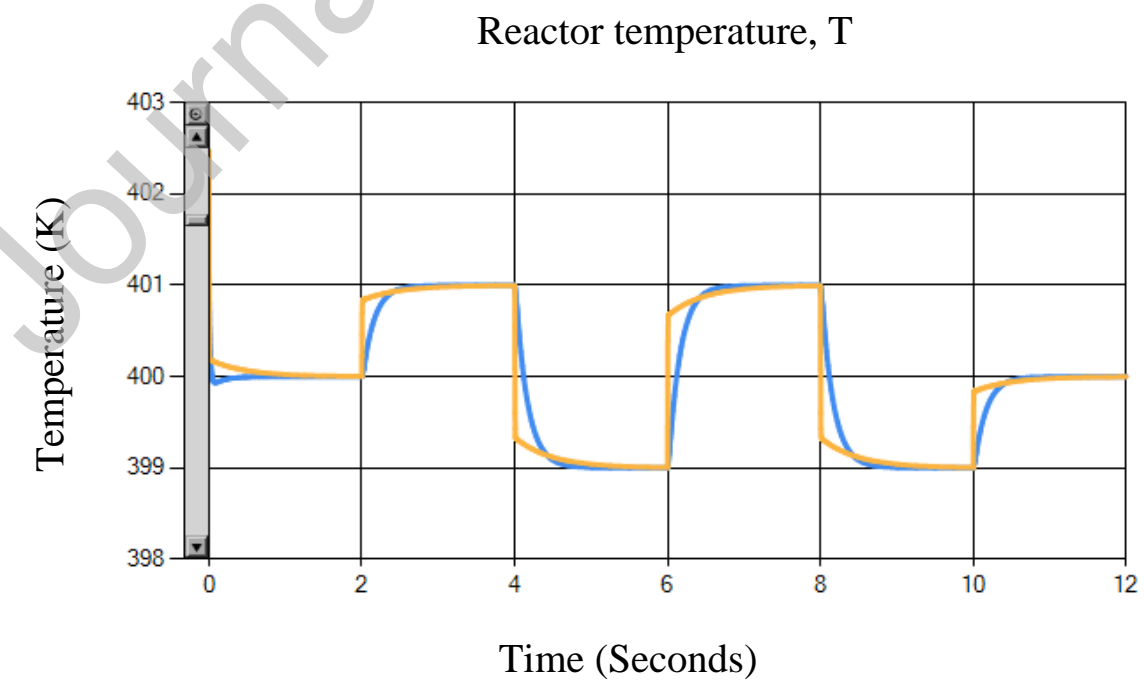

Figure 21: Comparison of set point tracking (Blue: initial values, Yellow: Optimized values) 


\subsection{Integrated Design and Control - II}

This application highlights the use of the integrated process design and control option in ProCACD (see section 3.5.1) through design and control of Methyl Tert Butyl Ether (MTBE) production using reactive distillation, studied earlier by Mansouri et al. (2016a) and many others. The decomposition approach for integrated design and control is used in this application example.

\section{Step 1: Problem definition}

The objective is to determine the reactive distillation design parameters (number of stages, feed location, reflux rate, etc.) as well as the controller pairing (two pairs of measured and controller variables) with the graphical method and compare the controller performance for other controller pairings. In this process methanol reacts with isobutene to produce MTBE in the presence of an acidic catalyst. The reaction is reversible and exothermic, with a heat of reaction of $-37.2 \mathrm{~kJ} / \mathrm{mol}$ in the liquid phase at $25^{\circ} \mathrm{C}$ (Al-Jarallah et al., 1988). Representing this reactive system in terms of elements (PerezCisneros et al., 1997) only two elements are needed to represent this ternary reactive system. Table 5 gives details of the reaction and the two elements. It is assumed that no inert compound is present in the system. The feed conditions for production of MTBE are taken from Sánchez-Daza et al. (Sánchez Daza et al., 2003) and they are given in Table 6.

Table 5: Element reaction for MTBE reactive system (without inert).

$$
\text { Isobutene }\left(\mathrm{C}_{4} \mathrm{H}_{8}\right)+\operatorname{Methanol}\left(\mathrm{CH}_{3} \mathrm{OH}\right) \leftrightarrow \operatorname{MTBE}\left(\mathrm{C}_{5} \mathrm{H}_{12} \mathrm{O}\right)
$$

Element definition: $\quad A=\mathrm{C}_{4} \mathrm{H}_{8} \quad B=\mathrm{CH}_{3} \mathrm{OH}$

Element reaction: $\quad A+B \leftrightarrow \mathbf{A B}$ 
Table 6: Design targets and product specifications for MTBE system

\begin{tabular}{|l|c|c|c|}
\hline Component & \multicolumn{3}{|l|}{ Molar composition } \\
\hline & Feed & Distillate & Bottom \\
\hline Isobutene $\left(\mathrm{C}_{4} \mathrm{H}_{8}\right)$ & 0.7 & 0.98 & - \\
\hline Methanol $\left(\mathrm{CH}_{4} \mathrm{O}\right)$ & 0.3 & - & more than 0.8 \\
\hline MTBE $\left(\mathrm{C}_{5} \mathrm{H}_{12} \mathrm{O}\right)$ & 0 & - & $\mathrm{kmol} / \mathrm{h}$; Feed temperature and \\
\hline $\begin{array}{l}\text { Methanol conversion: more than } 80 \% \text {; Feed flowrate: } 100 \mathrm{kP} \text {; degree of vaporization }(q): 0.795 \\
\text { pressure: } 300 \mathrm{~K} \text { and } 101.3 \mathrm{kPa}\end{array}$
\end{tabular}

Step 2: Calculate the driving force diagram and identify the maximum available driving force The graphical process design option in ProCACD for reactive distillation is used to calculate the driving force diagram for the established binary element system. ProCACD uses Equation 40 to convert the calculated reactive bubble point data to the driving force plot (Sánchez Daza et al., 2003). Figure 22 shows the driving force diagram for the binary element system related to MTBE production. In Equation $40 W_{i}^{v}$ and $W_{i}^{l}$ represent element composition in vapor and liquid phase of element i.

$D F=W_{i}^{v}-W_{i}^{l}=\frac{W_{i}^{l} \alpha_{i j}}{1+W_{i}^{l}\left(\alpha_{i j}-1\right)}-W_{i}^{l}$

Based on the product specifications given in Table 6, the operating area is selected from the reactive driving force plot (Figure 22). From the driving force diagram the elemental composition of Isobutene (A) in distillate $\left(W_{A, D t}^{l}\right)$ and bottoms $\left(W_{A, B m}^{l}\right)$ are identified as 0.99 and 0.5 respectively. The operating area is the area of the triangle formed by the two identified elemental composition points and the maximum point of the driving force curve on the y-axis. 


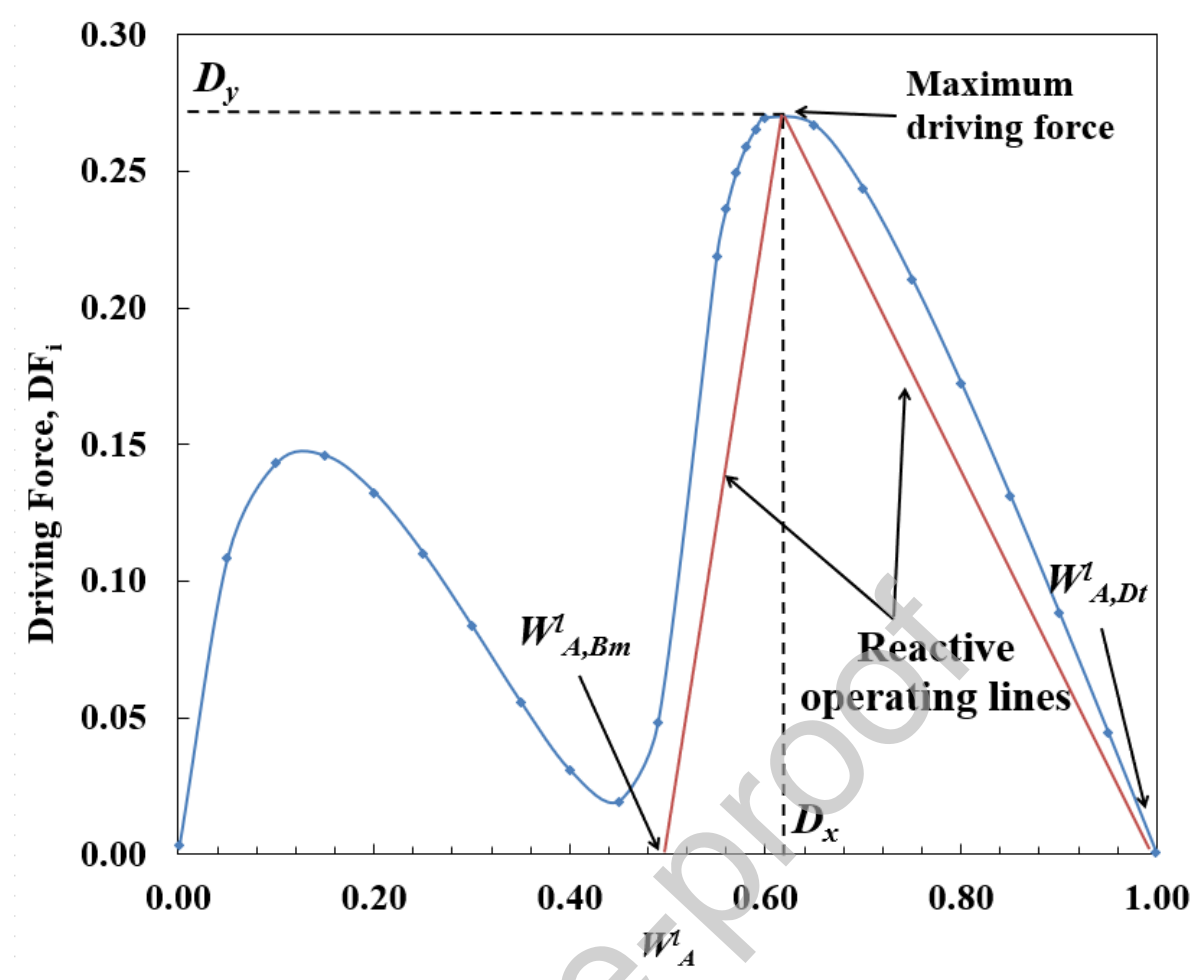

Figure 22: Reactive driving force diagram for MTBE reactive system $(\mathrm{P}=101.3 \mathrm{kPa})$ adopted from

(Mansouri et al., 2016a)

Step 3: Process design and controller pairing

Using the driving force diagram of Figure 22 and the design algorithm of Sanchez-Daza et al. (2003), the total number of stages is found to be 7 , with 5 reactive stages and 2 non-reactive stages, which are the partial reboiler and the total condenser. The optimal feed location is found to be stage 2 from the top of the column. The final reactive distillation column design configuration at the maximum driving force is shown in Figure 22.

For this reactive distillation column, the controlled variables include the light key element composition at the top and heavy key element composition at the bottom. While the manipulated variables include reflux ratio and reboiler duty. Using ProCACD, optimal controller pairing is verified through relative gain analysis as explained in section 3.3. Equation 41 and Table 7 show the transfer functions of the combinations of pairings that are possible for the two sets of manipulated and controlled variables (note that the light and heavy key element compositions from the simulation are converted to compositions of MTBE) corresponding to the design at the highest driving force. 
$G(s)=K \frac{1+\tau_{z} s}{\left(1+\tau_{p 1} s\right)\left(1+\tau_{p 2} s\right)}$

Table 7: Transfer function parameters

\begin{tabular}{|c|c|c|c|c|}
\hline Controlled variable/Manipulated variable & $\mathrm{K}$ & $\tau_{p 1}$ & $\tau_{p 2}$ & $\tau_{z}$ \\
\hline $\mathrm{X}^{\mathrm{B}}{ }_{\mathrm{MTBE}} / \mathrm{RR}$ & $0.32211[-]$ & 6.25 & 2.32 & -1.8092 \\
\hline $\mathrm{X}^{\mathrm{D}}{ }_{\mathrm{MTBE}} / \mathrm{RR}$ & $-4.96 \mathrm{E}-05[-]$ & 2 & 2 & 5.38 \\
\hline $\mathrm{X}^{\mathrm{B}}{ }_{\mathrm{MTBE}} / \mathrm{Q}_{\mathrm{R}}$ & $-1.23 \mathrm{E}-06[\mathrm{~kJ} / \mathrm{h}]$ & 3.7 & 3.68 & -0.64004 \\
\hline $\mathrm{X}^{\mathrm{D}}{ }_{\mathrm{MTBE}} / \mathrm{Q}_{\mathrm{R}}$ & $-1.47 \mathrm{E}-11[\mathrm{~kJ} / \mathrm{h}]$ & 0.02 & 0.02 & -601749 \\
\hline
\end{tabular}

With the gains shown in Table 7, we can calculate the relative gain array of the manipulated and controlled variables as follows.

$R G A=\left[\begin{array}{ccc} & \mathrm{X}_{\mathrm{MTBE}}^{\mathrm{B}} & \mathrm{X}_{\mathrm{MTBE}}^{\mathrm{D}} \\ R R & 0.072 & 0.928 \\ Q_{R} & 0.928 & 0.072\end{array}\right]$

From the relative gain analysis, it is clear that the best controller pairing is to control the light key element composition at the top with the reflux ratio (manipulated variable) and the heavy key element composition at the bottom with the reboiler duty. These results also match with the published results from Mansouri et al. (2016a). 


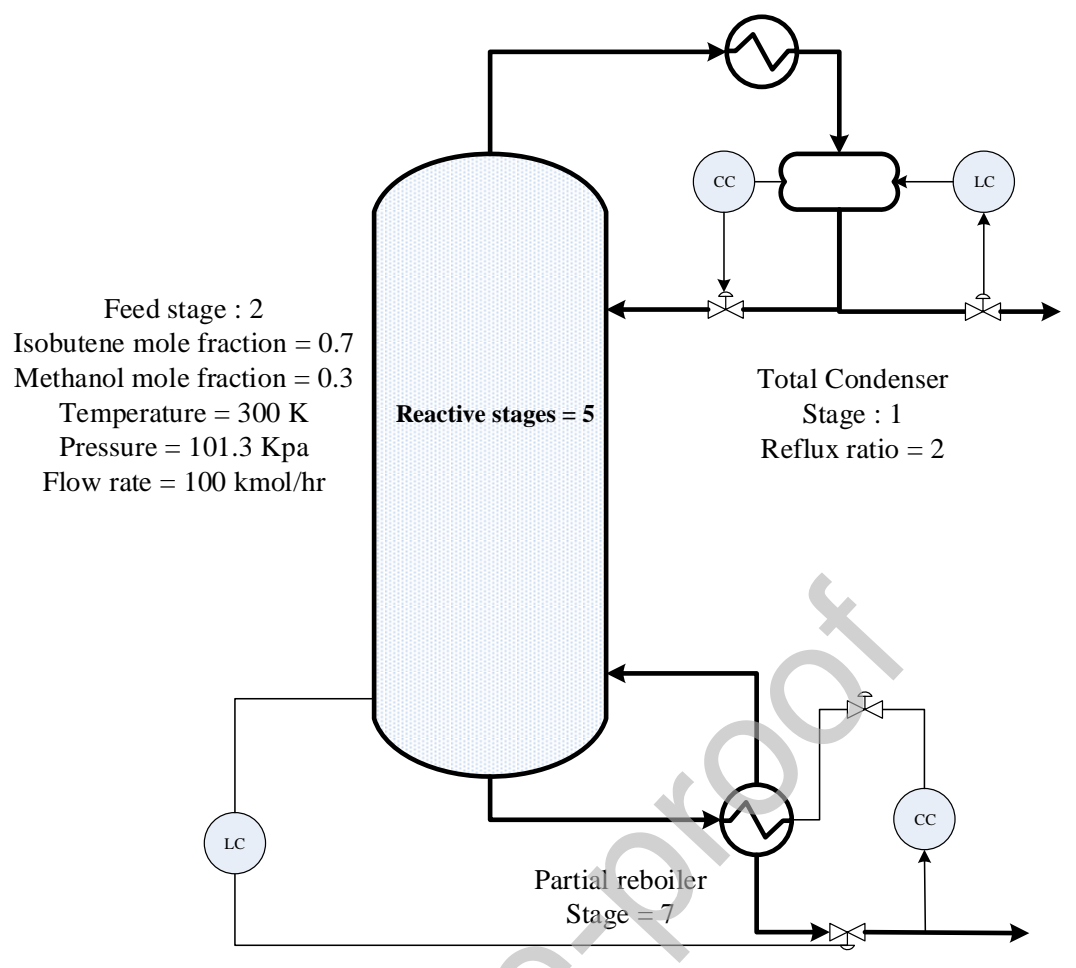

Figure 23: Reactive distillation column design for MTBE

Step 4: Controller design

Figure 23 presents the control configuration in which the purities of both the top and the bottom products are measured and controlled. This control structure implementation follows the relative gain array (RGA) analysis conducted in step 3, which showed that the composition of the MTBE in distillate is controlled by manipulating the reflux flow rate in the top control loop. In the bottom control loop, the composition of the MTBE in bottom is controlled by manipulating the heat duty of the reboiler. The levels of the reflux drum and the reboiler are controlled by the distillate and bottom product flow rates, respectively. Note however, in this case study, the level controllers are proportional (P) type which are preselected and they are included in the model equations for dynamic model consistency and stability. Furthermore, it is assumed that there is a perfect pressure control on the column and thus, the pressure changes in the column are neglected.

Step 5: Verification and comparison of performances

For this application example, Aspen dynamics which is integrated with ProCACD is used to run the closed-loop simulations based on the identified control structure from step 4. Figure 24 shows the 
closed- loop performance of optimal design-control solution for a disturbance in the feed. In this case the isobutene flow rate in the feed is changed from 75 to $85 \mathrm{kmol} / \mathrm{hr}$. Figures $24 \mathrm{a}$ and $24 \mathrm{~b}$ show the controller action for changes in the manipulated variables, that is, reflux ratio and reboiler duty (heat addition), respectively, to reject the disturbance in the feed and to maintain composition of MTBE in the distillate $(0.033 \%)$ and bottoms $(84 \%)$. From the results it can be observed that the design solution based on driving force-based method is not sensitive towards feed disturbances and could maintain the controlled variables at their original set-points with a relatively small effort in the manipulated variables. Similarly, several other case studies involving reaction-separation systems have been verified (details can be obtained from the authors).
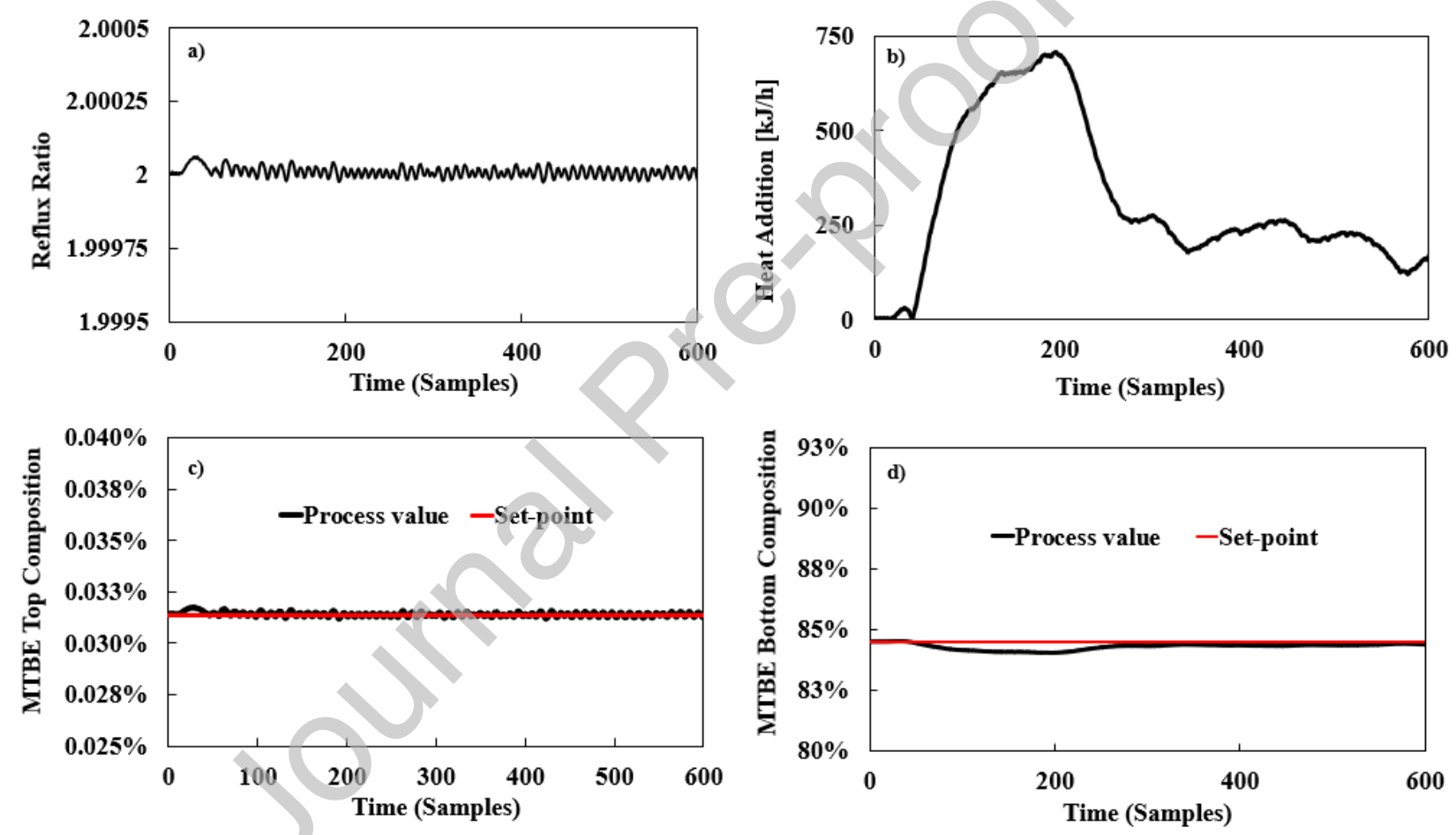

Figure 24: Closed-loop performance of optimal design-control solution (each time sample is 5 seconds). a) change in reflux ratio (MV) b) change in reboiler duty (MV) c) MTBE top composition d)

MTBE bottom composition

\section{Conclusion}

In this work, a computer-aided tool, ProCACD, that integrates process modeling, simulation, optimization, design and control in a unified platform to solve a wide range of control related problems 
has been presented. With built-in model library, analysis methods, design methods, and integrated tools, ProCACD enables users to study the system to design and verify different aspects of process control. The novelty and contribution of this work is the use of a components-based architecture to develop an efficient, flexible and systematic computer aided tool, which can be used for teaching and training purposes, for industrial problem solution as well as research purposes. While no new methods for integration of design and control has been proposed, verification and validation of several controller design and integration process-control design has been made. This feature allows the user to try out different design methods without too much work related to model development, data transfer and problem solution. The flexible architecture allows ProCACD to quickly implement new methods for controller design, or add new process models or new analysis techniques. Current version of ProCACD employs first-principles based models for the system (process). However, since many industrial applications require data-driven applications, current and future work will concentrate on developing and implementing these features in ProCACD. Also, a library for solved case studies involving a wide range of control related problems for a wide range of processing systems is under construction. Note that the objective has not been to develop new design and/or control techniques but to collect developed desing and control tools and apply them through ProCACD.

\section{Acknowledgments}

We gratefully acknowledge the financial support of the National Key Research and Development Program of China (No. 2017YFE0106700) and the National Natural Science Foundation of China (No. 61973268).

\section{Author Statement}

Anjan K. Tula: Software development, Data curation, Writing- Original draft Jialiang Wang: Software development

Xi Chen: Conceptualization, Methodology, Writing- Reviewing and Editing Seyed S. Mansouri: Partial development of Graphical design-based decomposition approach and application 4.4 Rafiqul Gani: Conceptualization, Methodology, Writing- Reviewing and Editing 


\section{References}

Al-Jarallah, A.M., Lee, A.K.K., Siddiqui, M.A.B., 1988. Kinetics of methyl tertiary butyl ether synthesis catalyzed by sulphuric acid. Chem. Eng. J. 39, 169-174.

Alvarado-Morales, M., Hamid, M.K.A., Sin, G., Gernaey, K. V., Woodley, J.M., Gani, R., 2010. A model-based methodology for simultaneous design and control of a bioethanol production process. Comput. Chem. Eng. 34, 2043-2061.

Aspen Technology, 2019a. Aspen Plus: User guide.

Aspen Technology, 2019b. Aspen HYSYS: User Guide.

Aspen Technology, 2019c. Aspen Dynamics: User guide.

Aspen Technology, 2019d. Aspen Custom Mode ler: User guide.

Cameron, I., Gani, R., 2011. Product and Process Modelling, Product and Process Modelling. Elsevier. Chemstations, 2019. Chemcad: User guide.

Cooper, D.J., 1996. Picles (TM): a simulator for "virtual world" education and training in process dynamics and control. Comput. Appl. Eng. Educ. 4, 207-215.

Dimian, A.C., Bildea, C.S., Kiss, A., n.d. Integrated design and simulation of chemical processes.

Fedorova, M., Sin, G., Gani, R., 2015. Computer-aided modelling template: Concept and application. Comput. Chem. Eng. 83, 232-247.

Gani, R., Bek-Pedersen, E., 2000. Simple new algorithm for distillation column design. AIChE J.

Gani, R., Hytoft, G., Jaksland, C., Jensen, A.K., 1997. An integrated computer aided system for integrated design of chemical processes. Comput. Chem. Eng. 21, 1135-1146.

Glasser, D., Hildebrandt, D., 1997. Reactor and process synthesis. Comput. Chem. Eng. 21.

Gollapalli, U., Dantus, M.M., High, K.A., 2000. Environment and control issues in design. Comput. Chem. Eng. 24, 1709-1712.

Hamid, M.K.A., Sin, G., Gani, R., 2010. Integration of process design and controller design for chemical processes using model-based methodology. Comput. Chem. Eng. 34, 683-699.

Hildebrandt, D., Glasser, D., 1990. The attainable region and optimal reactor structures. Chem. Eng. Sci. $45,2161-2168$.

Holt, B.R., Jerome, N.F., Buck, U., Dubinsky, M., Economou, C., Grimm, W., Galimidi, A., Grosdidier, P., Jordan, K., Klewin, R., Kraemer, W., Kuguenko, G. V., Mandler, J., Ness, A., Ninman, T.A., Plocher, T., Rivera, D.E., Sela, R., Szakaly, Z., Tung, H.H., Webb, C., Zafiriou, E., 
Morari, M., Ray, W.H., 1987. Consyd-Integrated software for computer aided control system design and analysis. Comput. Chem. Eng. 11, 187-203.

Horn.F, 1964. Attainable and non-attainable regions in chemical reaction technique. Proc. Third Eur. Symp. Chem. React. Eng. 293-303.

Howes, S., Mohler, I., Bolf, N., 2016. Real-time dynamic process control loop identification, tuning and optimization software. IFAC-PapersOnLine 49, 213-217.

Lopez-Arenas, T., Mansouri, S.S., Sales-Cruz, M., Gani, R., Pérez-Cisneros, E.S., 2019. A Gibbs energy-driving force method for the optimal design of non-reactive and reactive distillation columns. Comput. Chem. Eng. 128, 53-68.

Mansouri, S.S., Huusom, J.K., Gani, R., Sales-Cruz, M., 2016a. Systematic integrated process design and control of binary element reactive distillation processes. AIChE J. 62, 3137-3154.

Mansouri, S.S., Sales-Cruz, M., Huusom, J.K., Gani, R., 2016b. Systematic integrated process design and control of reactive distillation processes involving multi-elements. Chem. Eng. Res. Des. 115, $348-364$.

Marchetti, A., Chachuat, B., Bonvin, D., 2009. Modifier-Adaptation Methodology for Real-Time Optimization. Ind. Eng. Chem. Res. 48, 6022-6033.

Mathworks, 2019a. Matlab: Documentation.

Mathworks, 2019b. Simulink: Documentation.

Maurmaier, M., 2008. Leveraging model-driven development for automation systems development. In: IEEE International Conference on Emerging Technologies and Factory Automation, ETFA. pp. 733-737.

Nelson, D.A., Kirkwood, R.L., Douglas, J.M., 1989. Conceptual design of chemical processes. In: Simulation Series. McGraw-Hill, pp. 180-184.

Perez-Cisneros, E., Schenk, M., Gani, R., Pelavachi, P.A., 1996. Aspects of simulation, design and analysis of reactive distillation operations. Comput. Chem. Eng. 20.

PID Control Laboratory, n.d. pidlab.com [WWW Document].

Piela, P.C., 1990. ASCEND : an object-oriented computer environment for modeling and analysis. Carnegie Mellon University.

Pistikopoulos, E.N., Diangelakis, N.A., Oberdieck, R., Papathanasiou, M.M., Nascu, I., Sun, M., 2015. PAROC - An integrated framework and software platform for the optimisation and advanced 
model-based control of process systems. Chem. Eng. Sci. 136, 115-138.

Powell, M.J., 2009. The BOBYQA algorithm for bound constrained optimization without derivatives. Cambridge.

Rosenthal, R.E., 2007. GAMS-A User's Guide.

Sales-Cruz, M., Gani, R., 2003. A modelling tool for different stages of the process life. In: Computer Aided Chemical Engineering. Elsevier, pp. 209-249.

Sánchez Daza, O., Pérez-Cisneros, E.S., Bek-Pedersen, E., Gani, R., 2003. Graphical and Stage-toStage Methods for Reactive Distillation Column Design. AIChE J. 49, 2822-2841.

Seferlis, P., Georgiadis, M.C., 2004. The integration of process design and control. Elsevier.

SimSci, 2018. PRO/II: User guide.

Taiji-PID [WWW Document], 2019. URL www.taijipid.com/

Tula, A.K., Babi, D.K., Bottlaender, J., Eden, M.R., Gani, R., 2017. A computer-aided software-tool for sustainable process synthesis-intensification. Comput. Chem. Eng. 105, 74-95.

Tula, A.K., Eden, M.R., Gani, R., 2015. Process synthesis, design and analysis using a process-group contribution method. Comput. Chem. Eng. 81, 245-259.

Yuan, Z., Chen, B., Sin, G., Gani, R., 2012. State-of-the-art and progress in the optimization-based simultaneous design and control for chemical processes. AIChE J. 


\section{Declaration of interests}

The authors declare that they have no known competing financial interests or personal relationships that could have appeared to influence the work reported in this paper.

$\square$ The authors declare the following financial interests/personal relationships which may be considered as potential competing interests: 